Algebraic $8 \mathcal{G}$ Geometric $\mathcal{T}$ opology

Volume 4 (2004) 685-719

Published: 9 September 2004

ATG

\title{
Heegaard Floer homology of certain mapping tori
}

\author{
StANisLaV JABUKA \\ THOMAS MARK
}

\begin{abstract}
We calculate the Heegaard Floer homologies $\operatorname{HF}^{+}(M, \mathfrak{s})$ for mapping tori $M$ associated to certain surface diffeomorphisms, where $\mathfrak{s}$ is any $\operatorname{Spin}^{c}$ structure on $M$ whose first Chern class is non-torsion. Let $\gamma$ and $\delta$ be a pair of geometrically dual nonseparating curves on a genus $g$ Riemann surface $\Sigma_{g}$, and let $\sigma$ be a curve separating $\Sigma_{g}$ into components of genus 1 and $g-1$. Write $t_{\gamma}, t_{\delta}$, and $t_{\sigma}$ for the right-handed Dehn twists about each of these curves. The examples we consider are the mapping tori of the diffeomorphisms $t_{\gamma}^{m} \circ t_{\delta}^{n}$ for $m, n \in \mathbb{Z}$ and that of $t_{\sigma}^{ \pm 1}$.
\end{abstract}

AMS Classification 57R58; 53D40

Keywords Heegaard Floer homology, mapping tori

\section{Introduction}

In [11, Peter Ozsváth and Zoltán Szabó introduced a set of new invariants of 3-manifolds, the Heegaard Floer homology groups. There are several variations available in the construction, which give rise to several related invariants $H F^{+}(Y, \mathfrak{s}), H F^{-}(Y, \mathfrak{s}), H F^{\infty}(Y, \mathfrak{s})$, and $\widehat{H F}(Y, \mathfrak{s})$. Each of these is a relatively graded group associated to a closed oriented 3-manifold $Y$ equipped with a $\operatorname{Spin}^{c}$ structure $\mathfrak{s}$. This paper is concerned with the calculation of the group $\mathrm{HF}^{+}$in case $\mathrm{Y}$ is a fibered 3-manifold whose monodromy is of a particular form.

If $\Sigma_{g}$ is a closed oriented surface of genus $g>1$ and $\phi: \Sigma_{g} \rightarrow \Sigma_{g}$ is an orientation-preserving diffeomorphism, we can form the mapping torus $M(\phi)$ as a quotient of $\Sigma_{g} \times[0,1]$. For a simple closed curve $c$ on $\Sigma_{g}$, let $t_{c}: \Sigma_{g} \rightarrow \Sigma_{g}$ denote the right-handed Dehn twist about $c$. Choose a pair of geometrically dual nonseparating circles $\gamma$ and $\delta$ on $\Sigma_{g}$, and also let $\sigma$ be a circle that separates $\Sigma_{g}$ into components of genus 1 and $g-1$. Our main result is the calculation of $\mathrm{HF}^{+}(M, \mathfrak{s})$, where $M$ is any of the mapping tori $M\left(t_{\gamma}^{m} t_{\delta}^{n}\right)$ for $m, n \in \mathbb{Z}$ or $M\left(t_{\sigma}^{ \pm 1}\right)$ and $\mathfrak{s}$ is any non-torsion $\operatorname{Spin}^{c}$ structure on $M$. 
To state the results, recall that the homology group $H_{2}(M(\phi) ; \mathbb{Z})$ of the mapping torus $M(\phi)$ can be identified with $\mathbb{Z} \oplus \operatorname{ker}\left(1-\phi_{*}\right)$ where $\phi_{*}$ denotes the action of $\phi$ on $H_{1}(\Sigma ; \mathbb{Z})$. For a fixed integer $k$, the two requirements

(1) $\left\langle c_{1}(\mathfrak{s}),\left[\Sigma_{g}\right]\right\rangle=2 k$ and

(2) $\left\langle c_{1}(\mathfrak{s}),[T]\right\rangle=0$ for all classes $[T]$ coming from $H_{1}\left(\Sigma_{g}\right)$

specify the $\operatorname{Spin}^{c}$ structure $\mathfrak{s}$ uniquely modulo torsion. Let $\mathcal{S}_{k} \subset \operatorname{Spin}^{c}(M(\phi))$ denote the collection of $\operatorname{Spin}^{c}$ structures satisfying these two conditions: then for $\mathfrak{s} \in \mathcal{S}_{k}$ the adjunction inequality for Heegaard Floer homology shows that $H F^{+}(M(\phi), \mathfrak{s})=0$ unless $|k| \leq g-1$.

Now let $X(g, d)$ denote the $\mathbb{Z}$-graded group whose value in degree $j$ is the homology group $H_{g-j}\left(\operatorname{Sym}^{d}\left(\Sigma_{g}\right) ; \mathbb{Z}\right)$ of the $d$-th symmetric power of $\Sigma_{g}$. By convention, $X(g, d)=0$ if $d<0$. We denote by $X(g, d)[m]$ the graded group $X(g, d)$ with the grading shifted up by $m$, and if $G$ is a group we let $G_{(m)}$ denote the graded group $G$ concentrated in grading $m$.

Theorem 1.1 Fix an integer $k$ with $0<|k| \leq g-1$, and let $n$ be a nonzero integer. Define $d=g-1-|k|$. For the mapping torus $M\left(t_{\gamma}^{n}\right)$ of the composition of $n$ right-handed Dehn twists about a nonseparating circle $\gamma \subset \Sigma_{g}$, there is a unique $\operatorname{Spin}^{c}$ structure $\mathfrak{s}_{k} \in \mathcal{S}_{k}$ for which we have an isomorphism of relatively graded groups

$H F^{+}\left(M\left(t_{\gamma}^{n}\right), \mathfrak{s}_{k}\right) \cong\left(X(g-1, d-1) \otimes H^{*}\left(S^{1} ; \mathbb{Z}\right)\right)[\varepsilon(n)] \oplus \Lambda^{2 g-2-d} H^{1}\left(\Sigma_{g-1}\right)_{(g-d)}$.

Here $\varepsilon(n)=0$ if $n>0$ and $\varepsilon(n)=-1$ if $n<0$.

For any other $\mathfrak{s}^{\prime} \in \mathcal{S}_{k}$, we have an isomorphism

$$
H F^{+}\left(M\left(t_{\gamma}^{n}\right), \mathfrak{s}^{\prime}\right) \cong X(g-1, d-1) \otimes H^{*}\left(S^{1} ; \mathbb{Z}\right) .
$$

Theorem 1.2 For $k$ and $d$ as above, let $m, n$ be nonzero integers. Then for the mapping torus $M\left(t_{\gamma}^{m} t_{\delta}^{n}\right)$ there is a unique $\operatorname{Spin}^{c}$ structure $\mathfrak{s}_{k} \in \mathcal{S}_{k}$ for which there is an isomorphism of relatively graded groups

$$
H F^{+}\left(M\left(t_{\gamma}^{m} t_{\delta}^{n}\right), \mathfrak{s}_{k}\right) \cong\left\{\begin{array}{l}
X(g-1, d-1) \oplus \Lambda^{2 g-2-d} H^{1}\left(\Sigma_{g-1}\right)_{(g-1-d)} \\
X(g-1, d-1)[-1] \oplus \Lambda^{2 g-2-d} H^{1}\left(\Sigma_{g-1}\right)_{(g-1-d)} \\
X(g-1, d-1)[-2] \oplus \Lambda^{2 g-2-d} H^{1}\left(\Sigma_{g-1}\right)_{(g-1-d)}
\end{array}\right.
$$

The three lines on the right-hand side above correspond to the three cases $m, n>0, m \cdot n<0$ and $m, n<0$ respectively. For all other $\mathfrak{s}^{\prime} \in \mathcal{S}_{k}$ (and regardless of the signs of $m$ and $n$ ) we have an isomorphism

$$
H F^{+}\left(M\left(t_{\gamma}^{m} t_{\delta}^{n}\right), \mathfrak{s}^{\prime}\right) \cong X(g-1, d-1) .
$$


For the case of the separating curve $\sigma \subset \Sigma_{g}$, there is no torsion in the second cohomology. Thus there is a unique $\operatorname{Spin}^{c}$ structure $\mathfrak{s}_{k} \in \mathcal{S}_{k}$, and we have

Theorem 1.3 Let $n$ be either 1 or -1 and let $k$ and $d$ be as above. Then for a genus-1 separating curve $\sigma$, we have an isomorphism of relatively graded groups

$H F^{+}\left(M\left(t_{\sigma}^{n}\right), \mathfrak{s}_{k}\right) \cong\left(X(g-1, d-1) \otimes H^{*}\left(S^{1} \sqcup S^{1}\right)\right)[\varepsilon(n)] \oplus \Lambda^{2 g-2-d} H^{1}\left(\Sigma_{g-1}\right)_{(g-d)}$,

where $\varepsilon(n)$ is as in theorem 1.1

Remark 1.4 (1) When $\mathfrak{s}$ is not a torsion Spin $^{c}$ structure, the Heegaard Floer homology group $\mathrm{HF}^{+}(M, \mathfrak{s})$ carries only a relative cyclic grading. Thus the above results should be interpreted as asserting the existence of a $\mathbb{Z}$ grading on the relevant Floer homology groups such that the stated isomorphisms hold.

(2) If $\mathfrak{s} \notin \mathcal{S}_{k}$ for some $k$, then for $\phi$ one of the diffeomorphisms considered above we have $H^{+}(M(\phi), \mathfrak{s})=0$ by the adjunction inequality: the homology of $M(\phi)$ is spanned by $\Sigma_{g}$ and classes represented by tori. The above theorems therefore give a complete calculation of $\mathrm{HF}^{+}(M(\phi))$ in nontorsion Spin $^{c}$ structures in the cases listed.

(3) The results of theorems 1.111.3 are sharpened slightly in theorems 4.3. 5.3, 5.7, and 6.3. In particular, we determine the "special" Spinc structure $\mathfrak{s}_{k}$ precisely, and in some cases we also describe the action of $H_{1}(M ; \mathbb{Z}) \otimes_{\mathbb{Z}} \mathbb{Z}[U]$ on $H^{+}(M ; \mathfrak{s})$.

As an application of theorems 1.111.3, we make the following observation on the genera of the possible fibration structures on $Y$, where $Y$ is one of the mapping tori considered in the theorems above. We should remark that the following result can also be deduced from the relation $\|\cdot\|_{A} \leq\|\cdot\|_{T}$ between the Alexander and Thurston norms on $H^{1}(Y ; \mathbb{Z})$ due to McMullen [5], together with the relationships between the Euler characteristic of $\mathrm{HF}^{+}$, the Seiberg-Witten invariant, and the Alexander polynomial. We give a proof based exclusively on Heegaard Floer homology.

Theorem 1.5 Let $Y$ denote the mapping torus of one of the diffeomorphisms $t_{\gamma}^{m} t_{\delta}^{n}$ or $t_{\sigma}^{ \pm 1}$ of the surface $\Sigma_{g}$ of genus $g \geq 2$ as above (including the identity diffeomorphism), and suppose $f: Y \rightarrow S^{1}$ is a fibration of $Y$ having as fiber the connected surface $F$. Then the genus of $F$ is of the form $g+n(g-1)$ for some $n \geq 0$. 
Proof In 13, Ozsváth and Szabó show that if $Y$ is a 3-manifold that fibers over the circle with connected fiber $F$ of genus $h>1$, then there is a unique $\operatorname{Spin}^{c}$ structure $\mathfrak{s}$ on $Y$ satisfying the following conditions:

(1) $\left\langle c_{1}(\mathfrak{s}),[F]\right\rangle=2 h-2$, and

(2) $H^{+}(Y, \mathfrak{s}) \neq 0$.

Furthermore, they prove that for this "canonical" $\operatorname{Spin}^{c}$ structure, $\mathrm{HF}^{+}(Y, \mathfrak{s}) \cong$ $\mathbb{Z}$. Theorem 1.5 follows from this together with the observation that according to theorems 1.1 1.3. there is only one possible canonical Spin ${ }^{c}$ structure. To see this, note that if $Y$ is $M\left(t_{\gamma}^{n}\right)$ for some $n \in \mathbb{Z}$ or $M\left(t_{\sigma}^{ \pm 1}\right)$ then there is only one $\operatorname{Spin}^{c}$ structure (up to conjugation) having $H F^{+}$equal to $\mathbb{Z}$, namely $\mathfrak{s}_{g-1}$. If $Y$ is $M\left(t_{\gamma}^{m} t_{\delta}^{n}\right)$ there may be more such $\operatorname{Spin}^{c}$ structures, but note that since all elements of $\mathcal{S}_{k}$ differ by torsion classes, all the Chern classes of $\operatorname{Spin}^{c}$ structures in that set have the same pairing with every homology class $[F] \in H_{2}(Y ; \mathbb{Z})$. Hence the uniqueness of the $\operatorname{Spin}^{c}$ structure satisfying (1) and (2) above implies that if $\mathcal{S}_{k}$ contains a canonical Spin ${ }^{c}$ structure then it contains only that $\operatorname{Spin}^{c}$ structure. This with an examination of theorem 1.2 rules out all $k$ except $k=g-1$, so $\mathfrak{s}_{g-1}$ is the only possible canonical $\operatorname{Spin}^{c}$ structure.

Now suppose $f: Y \rightarrow S^{1}$ is a fibration with connected fiber $F$, where $Y=\Sigma_{g} \times$ $[0,1] / \sim$ is a mapping torus as in the statement. The canonical $\operatorname{Spin}^{c}$ structure $\mathfrak{s}_{g-1}$ on $Y$ has $\left\langle c_{1}\left(\mathfrak{s}_{g-1}\right),\left[\Sigma_{g}\right]\right\rangle=2 g-2$, and $\left\langle c_{1}\left(\mathfrak{s}_{g-1}\right),[T]\right\rangle=0$ for all classes $[T] \in H_{2}(Y ; \mathbb{Z})$ coming from $\operatorname{ker}\left(1-\phi_{*}\right)$. It follows that the image of $c_{1}\left(\mathfrak{s}_{g-1}\right)$ under the natural homomorphism

$$
\rho: H^{2}(Y ; \mathbb{Z}) \rightarrow \operatorname{Hom}\left(H_{2}(Y ; \mathbb{Z}), \mathbb{Z}\right)
$$

is divisible by $2 g-2$. From the remarks above, we know $\mathfrak{s}_{g-1}$ is also the canonical $\operatorname{Spin}^{c}$ structure for the fibration $f$ : in particular we must have $\left\langle c_{1}\left(s_{g-1}\right),[F]\right\rangle=2 h-2$, where $h$ is the genus of $F$. Hence $2 h-2$ is divisible by $2 g-2$, which is equivalent to the statement that $h=g+n(g-1)$ for some $n \geq 0$.

Remark 1.6 We implicitly assume here that $h \geq 2$. However, if a 3-manifold $Y$ admits a fibration with fiber genus 1 - ie, $Y$ is a torus bundle over $S^{1}$-then $\mathrm{H}_{2}(Y ; \mathbb{Z})$ is generated by classes represented by tori. It then follows from the adjunction inequality that any $\operatorname{Spin}^{c}$ structure having nontrivial $\mathrm{HF}^{+}$must be torsion, so from theorems 1.1 1.3 none of the examples under consideration can admit such a structure. 
It is not hard to construct fibrations of genus $g+n(g-1)$ on $Y$ as above for any $n \geq 0$ : see for example [16, [7]. In fact, for any class $[F]$ with $\left\langle c_{1}\left(\mathfrak{s}_{g-1}\right),[F]\right\rangle \neq 0$ there is a fibration of $Y$ having fiber with homology class $[F]$ (see [15]).

It is interesting to compare the results of theorems 1.11.3 to Floer homologies of different flavours (see for example [1], 3, 14]). Specifically, Eaman Eftekhary 1] and Paul Seidel [14] have calculated the Lagrangian Floer homology $H F(f)$ of a surface diffeomorphism $f$ obtained by Dehn twists along an arrangement of curves $C=C_{1}^{+} \cup \ldots \cup C_{r}^{+} \cup C_{1}^{-} \cup \ldots \cup C_{s}^{-} \subset \Sigma_{g}$ (the superscripts indicate the handedness of the Dehn twists performed). Their results show that

$$
H F(f) \cong H^{*}\left(\Sigma_{g} \backslash\left(\cup_{i} C_{i}^{-}\right), \cup_{j} C_{j}^{+}\right)
$$

Our formulas from theorems 1.11.3 agree with the above when $k=g-2$.

Corollary 1.7 With the notation and hypotheses as in theorems 1.1 1.3, there are isomorphisms of relatively graded groups

$$
\begin{aligned}
H F^{+}\left(M\left(t_{\gamma}^{n}\right), \mathfrak{s}_{g-2}\right) & = \begin{cases}H^{*}\left(\Sigma_{g} \backslash \gamma\right) & n=-1 \\
H^{*}\left(\Sigma_{g}, \gamma\right) & n=1\end{cases} \\
H F^{+}\left(M\left(t_{\gamma}^{m} t_{\delta}^{n}\right), \mathfrak{s}_{g-2}\right) & = \begin{cases}H^{*}\left(\Sigma_{g}, \gamma \cup \delta\right) & m=n=1 \\
H^{*}\left(\Sigma_{g} \backslash \gamma, \delta\right) & m \cdot n=-1 \\
H^{*}\left(\Sigma_{g} \backslash(\gamma \cup \delta)\right) & m=n=-1\end{cases} \\
H F^{+}\left(M\left(t_{\sigma}^{n}\right), \mathfrak{s}_{g-2}\right) & = \begin{cases}H^{*}\left(\Sigma_{g} \backslash \sigma\right) & n=-1 \\
H^{*}\left(\Sigma_{g}, \sigma\right) & n=1\end{cases}
\end{aligned}
$$

The corollary follows from theorems 1.1 1.3 by direct calculation. For example, theorem 1.1 gives $H F^{+}\left(M\left(t_{\gamma}\right), \mathfrak{s}_{g-2}\right) \cong \mathbb{Z}_{(g-1)}^{2 g-1} \oplus \mathbb{Z}_{(g)}$ which is up to degree shift isomorphic to $H^{*}\left(\Sigma_{g}, \gamma\right)$.

Likewise, it is not hard to see using theorem 1.1 that $H F^{+}\left(M\left(t_{\gamma}\right), \mathfrak{s}_{k}\right)$ agrees with the periodic Floer homology $H P_{*}$ of Hutchings and Sullivan [3, in cases where the latter has been calculated. In particular, compare theorem 1.1 above with theorem 5.3 of 3 .

It is also worth comparing our results to the Seiberg-Witten invariants of the mapping tori. Recall that the Euler characteristic $\chi\left(H F^{+}(M, \mathfrak{s})\right)$ calculates the Seiberg-Witten invariant $S W_{M}(\mathfrak{s})$ (this follows from results in 10 together with [6]). Theorem 1.1 together with the result $H F^{+}\left(M(\mathrm{id}), \mathfrak{s}_{k}\right) \cong X(g, d)$ from [9] (where $k \neq 0$ and $d=g-1-|k|$ ) give the following observation: 
Corollary 1.8 The Seiberg-Witten invariants of the mapping tori $M$ (id) and $M\left(t_{\gamma}^{n}\right)$ with $0 \neq n \in \mathbb{Z}$ in the spin ${ }^{c}$-structure $\mathfrak{s}_{k}$ are all equal to $\pm\left(\begin{array}{c}2 g-2 \\ g-1-|k|\end{array}\right)$ while the Heegaard Floer homology groups in these Spin ${ }^{c}$ structures are mutually nonisomorphic when $|k| \neq g-1$.

Note that the Euler characteristics of the groups $H F^{+}\left(M(\phi), \mathfrak{s}^{\prime}\right)$, for $\phi$ as in the corollary and $\mathfrak{s}_{k} \neq \mathfrak{s}^{\prime} \in \mathcal{S}_{k}$, are all zero.

Proof In section 4 we show that $H F^{+}\left(M\left(t_{\gamma}^{n}\right), \mathfrak{s}_{k}\right)$ is calculated as the homology of $(X(g, d), D)$ where $D$ is a certain differential on $X(g, d)$ (which is described in detail in section 3). In section B we establish an isomorphism of $X(g, d)$ with $X_{+}(g, d) \oplus X(g-1, d-1) \oplus X(g-1, d-1)$ and show that of the last two summands, only the first one consists of cycles for $D$. Thus the total rank of $H F^{+}\left(M\left(t_{\gamma}^{n}\right), \mathfrak{s}_{k}\right)$ is less than that of $H F^{+}\left(M(\mathrm{id}), \mathfrak{s}_{k}\right)=X(g, d)$ by at least the rank of $X(g-1, d-1)$. The latter has positive rank precisely when $d-1 \geq 0$ or equivalently when $|k| \leq g-2$.

The main tools used to prove theorems 1.1] 1.3 are introduced in 9], where Ozsváth and Szabó extend their theory to produce invariants of nullhomologous knots in 3-manifolds. In the same article they developed a technique (which we describe in some detail in section 2) that under favorable circumstances allows one to calculate the Heegaard Floer homology of a manifold obtained as the zero framed surgery along a knot $K$ in a 3-manifold $Y$. Ozsváth and Szabó used this technique to find the Floer homology groups of the mapping tori associated to the identity map and also the diffeomorphism induced by a single Dehn twist along a non-separating curve (though in the latter case the result is not presented explicitly). The proofs of the theorems above follow the outline of those calculations.

The remainder of the paper is organized as follows. In section 2 we review the construction from [9] which explains how to calculate $\mathrm{HF}^{+}\left(Y_{0}(K)\right)$ from $H F^{+}(Y)$ and a certain quotient complex of the "knot complex" $C F K^{\infty}(Y, K)$. In section 3 we introduce some auxiliary notation that is used in subsequent sections and also give a model calculation for $\mathrm{HF}^{+}\left(M\left(t_{\gamma}\right)\right)$ that sets the agenda for later sections. In sections 4 , 5] and 6] we calculate the Heegaard Floer homologies of $M\left(t_{\gamma}^{n}\right), M\left(t_{\gamma}^{m} t_{\delta}^{n}\right)$ and $M\left(t_{\sigma}^{ \pm 1}\right)$ respectively.

Acknowledgements We would like to thank Peter Ozsváth for useful conversations and encouragement, and Ron Fintushel for referring us to [16]. 


\section{Calculating the Heegaard Floer homology of the zero surgery}

In this section we gather some general results that are used in our calculations in the subsequent sections. The results presented here are an adaptation of the techniques developed in [9], tailored for the applications we have in mind. The main theorem of this section (theorem 2.1 below) explains how one can calculate the Heegaard Floer homology $\mathrm{HF}^{+}$of the manifold $Y_{0}(K)$ obtained from zero surgery along a nullhomologous knot $K$ in $Y$. Recall that a knot $K \subset Y$ gives rise to a refined version of Heegaard Floer homology, whose construction we now summarize. The reader is referred to [9] for more details.

Given $K$, let $E$ denote the torus boundary of a regular neighborhood of $K$. One can then find a Heegaard surface for $Y$ of the form $E \# \Sigma_{g-1}$, with attaching circles $\boldsymbol{\alpha}=\alpha_{1}, \ldots, \alpha_{g}$ and $\boldsymbol{\beta}=\beta_{1}, \ldots, \beta_{g}$. Fix a meridian for $K$ lying on $E$, and let $w$ and $z$ denote a pair of basepoints, one on each side of this meridian. The data $(E \# \Sigma, \boldsymbol{\alpha}, \boldsymbol{\beta}, w)$ together with a choice of $\operatorname{Spin}^{c}$ structure $\mathfrak{s}$ on $Y$ can be used to define the Heegaard Floer chain groups $C F^{\infty}(Y, \mathfrak{s})$. The additional basepoint $z$, along with a choice of "relative $\operatorname{Spin}^{c}$ structure" $\mathfrak{s} \in \operatorname{Spin}^{c}\left(Y_{0}(K)\right)$ lifting $\mathfrak{s}$ gives rise to a filtration $\mathcal{F}$ on $C F^{\infty}(Y, \mathfrak{s})$. The "knot chain complex" $C F K^{\infty}(Y, K, \underline{\mathfrak{s}})$ is this filtered complex.

More concretely, we assume that $K$ is nullhomologous and fix a Seifert surface $F$ for $K$. Then $F$ specifies the zero-framing on $K$, and can be capped off to a closed surface $\hat{F}$ in the zero-surgery $Y_{0}(K)$. The generators of $C F K^{\infty}(Y, K, \underline{\mathfrak{s}})$ are triples $[\mathbf{x}, i, j]$, where $\mathbf{x}$ denotes an intersection point between the tori $T_{\alpha}=\alpha_{1} \times \cdots \times \alpha_{g}$ and $T_{\beta}=\beta_{1} \times \cdots \times \beta_{g}$ in the symmetric power $\operatorname{Sym}^{g}(E \# \Sigma)$, and $i$ and $j$ are integers. The point $\mathbf{x}$ along with the basepoint $w$ determine a $\operatorname{Spin}^{c}$ structure $\mathfrak{s}_{w}(\mathbf{x})$ on $Y$ as well as a relative $\operatorname{Spin}^{c}$ structure $\underline{\mathfrak{s}}_{w}(\mathbf{x})$; we require that $\mathfrak{s}_{w}(\mathbf{x})=\mathfrak{s}$. Furthermore, $i$ and $j$ are required to satisfy the equation

$$
\left\langle c_{1}\left(\underline{\mathfrak{s}}_{w}(\mathbf{x})\right),[\hat{F}]\right\rangle=2(j-i) .
$$

In this notation, the filtration $\mathcal{F}$ is simply $\mathcal{F}([\mathbf{x}, i, j])=j$; changing the Seifert surface $F$ shifts $\mathcal{F}$ by a constant.

The boundary map $\partial^{\infty}$ in $C F K^{\infty}$ is defined by counting holomorphic disks in $\operatorname{Sym}^{g}(E \# \Sigma)$ and can only decrease the integers $i$ and $j$. Thus, for example, the subgroup $C\{i<0\}$ of $C F K^{\infty}$ generated by those $[\mathbf{x}, i, j]$ having $i<0$ is a subcomplex, and indeed is simply $C F^{-}(Y, \mathfrak{s})$ with an additional filtration. The quotient of $C F K^{\infty}$ by $C\{i<0\}$ is written $C\{i \geq 0\}$, and is a filtered 
version of $C F^{+}(Y, \mathfrak{s})$. We will use other similar notations to indicate other sub- or quotient complexes of $C F K^{\infty}$. In particular, $\widehat{C F K}$ is by definition the quotient complex $C\{i=0\}$, and $\widehat{H F K}$ is the homology of the graded object associated to the filtration $\mathcal{F}$ of $\widehat{C F K}$. We denote by $\widehat{H F K}(Y, K ; j)$ the summand of this group supported in filtration level $j$ (typically suppressing the $\operatorname{Spin}^{c}$ structure from the notation).

As an additional piece of structure, we have a natural chain endomorphism $U$ on $C F K^{\infty}$ given by $U:[\mathbf{x}, i, j] \mapsto[\mathbf{x}, i-1, j-1]$.

One of the main calculational tools available in Floer homology is the long exact sequence in homology arising from a triple of manifolds $Y, Y_{0}$, and $Y_{n}$. Here $Y_{0}$ and $Y_{n}$ denote the results of 0 - or $n$-framed surgery on a nullhomologous knot $K$ in $Y$. In this situation, Ozsváth and Szabó define a surjective map $Q: \operatorname{Spin}^{c}\left(Y_{0}\right) \rightarrow \operatorname{Spin}^{c}\left(Y_{n}\right)$ (see section 9 of [10]), and set

$$
H F^{+}\left(Y_{0}, Q^{-1}(\mathfrak{t})\right)=\bigoplus_{Q(\mathfrak{s})=\mathfrak{t}} H F^{+}\left(Y_{0}, \mathfrak{s}\right)
$$

Furthermore, if we let $W_{n}$ denote the cobordism connecting $Y_{n}$ with $Y$ comprising a single 2-handle addition, then a Seifert surface $F$ for $K$ can be completed to a surface $\tilde{F}$ in $W_{n}$. Then given a $\operatorname{Spin}^{c}$ structure $\mathfrak{s}$ on $Y$ and an integer $k$, we can specify a $\operatorname{Spin}^{c}$ structure $\mathfrak{s}_{k}$ on $Y_{n}$ by requiring that $\mathfrak{s}_{k}$ be cobordant to $\mathfrak{s}$ by a $\operatorname{Spin}^{c}$ structure $\mathfrak{r}$ on $W_{n}$ having $\left\langle c_{1}(\mathfrak{r}),[\tilde{F}]\right\rangle=n+2 k$.

The theorem we're aiming for in this section is:

Theorem 2.1 Let $K$ be a nullhomologous knot in $Y$, and assume that for all sufficiently large positive integers $n, H F_{r e d}^{+}(Y)=H F_{r e d}^{+}\left(Y_{n}\right)=0$. Fix a positive integer $k$. Then there is a grading on $H F^{+}\left(Y_{0}, Q^{-1}\left(\mathfrak{s}_{k}\right)\right)$ as a $\Lambda^{*} H_{1}\left(Y_{0}\right) \otimes_{\mathbb{Z}} \mathbb{Z}[U]$-module for which there is an isomorphism

$$
H F^{+}\left(Y_{0}, Q^{-1}\left(\mathfrak{s}_{k}\right)\right) \cong H_{*}(C\{i<0 \text { and } j \geq k\})
$$

of graded $\mathbb{Z}$-modules. The case of $k<0$ is handled by conjugation duality of $\mathrm{HF}^{+}$(see comment below).

Note that if $\mathfrak{s}_{k}=(\mathfrak{s}, k) \in \operatorname{Spin}^{c}\left(Y_{n}\right)$ is determined by $\mathfrak{s} \in \operatorname{Spin}^{c}(Y)$ and $k \in \mathbb{Z}$, then the dual spin $^{c}$-structure $\overline{\mathfrak{s}_{k}}$ is given by $\overline{\mathfrak{s}_{k}}=(\overline{\mathfrak{s}},-k)=\overline{\mathfrak{s}}_{-k}$. On the other hand, the map $Q: \operatorname{Spin}^{c}\left(Y_{0}\right) \rightarrow \operatorname{Spin}^{c}\left(Y_{n}\right)$ is equivariant with respect to conjugation, specifically

$$
Q^{-1}\left(\mathfrak{s}_{-k}\right)=\overline{Q^{-1}\left(\overline{\mathfrak{s}}_{k}\right)}
$$


The invariance of $\mathrm{HF}^{+}$under conjugation of spin $^{c}$-structures together with theorem 2.1 now gives

$$
H F^{+}\left(Y_{0}, Q^{-1}\left(\mathfrak{s}_{-k}\right)\right) \cong H F^{+}\left(Y_{0}, Q^{-1}\left(\overline{\mathfrak{s}}_{k}\right)\right)
$$

In what follows we will thus focus on the case $k>0$, the case $k<0$ is handled by equation (11).

Notice furthermore that by choosing $n$ sufficiently large, we can assume that $Q^{-1}\left(\mathfrak{s}_{k}\right)$ consists of a single $\operatorname{spin}^{c}$-structure with nonvanishing $H F^{+}$. The reason for this is that under the identification $\operatorname{Spin}^{c}\left(Y_{0}\right) \cong \operatorname{Spin}^{c}(Y) \oplus \mathbb{Z}$, any two $\operatorname{spin}^{c}$-structures $\mathfrak{t}_{1}, \mathfrak{t}_{2} \in Q^{-1}\left(\mathfrak{s}_{k}\right)$ differ by $(0,2 n)$. Since $Y_{0}$ has only finitely many $\operatorname{spin}^{c}$-structure $\mathfrak{t}$ for which $H^{+}\left(Y_{0}, \mathfrak{t}\right) \neq 0$, the assertion follows.

We also consider conditions under which the isomorphism from theorem 2.1 is an isomorphism of $\Lambda^{*} H_{1}\left(Y_{0}\right) \otimes \mathbb{Z}[U]$ modules: see theorem 2.5] below.

We should note that this theorem was proved in 9 for the case relevant to the mapping tori $M(\mathrm{id})$ and $M\left(t_{\gamma}\right)$, and that the proof given here is little more than a summary of that one.

\section{$2.1 \quad H F^{+}\left(Y_{0}(K)\right)$ as a $\mathbb{Z}$-module}

We proceed to the proof of theorem 2.1.

As above, a choice of integer $k$ gives rise to a $\operatorname{Spin}^{c}$ structure $\mathfrak{s}_{k}$ on $Y_{n}$. In what follows, we will write $C F^{+}\left(Y_{n},[k]\right)$ for $C F^{+}\left(Y_{n}, \mathfrak{s}_{k}\right)$, and often suppress the $\operatorname{Spin}^{c}$ structure entirely from the notation for the knot complex. In section 4 of 9], Ozsváth and Szabó prove the following theorem which calculates $H F^{+}\left(Y_{n}\right)$ in terms of the homology of a quotient complex of $C F K^{\infty}(Y, K)$.

Theorem 2.2 (Theorem 4.4 of [9]) For all $n$ sufficiently large, there exists an isomorphism of chain complexes

$$
{ }^{b} \Psi^{+}: C F^{+}\left(Y_{n},[k]\right) \rightarrow C\{i \geq 0 \text { or } j \geq k\}
$$

We proceed by considering the surgery long exact sequence for $Y \rightarrow Y_{0} \rightarrow Y_{n}$ for $n$ chosen sufficiently large so that theorem 2.2 applies. The sequence in question is

$$
\cdots \stackrel{F}{\longrightarrow} H F^{+}(Y) \stackrel{G}{\longrightarrow} H F^{+}\left(Y_{0}, Q^{-1}([k])\right) \stackrel{H}{\longrightarrow} H F^{+}\left(Y_{n},[k]\right) \stackrel{F}{\longrightarrow} \cdots
$$

where $Q: \operatorname{Spin}^{c}\left(Y_{0}\right) \rightarrow \operatorname{Spin}^{c}\left(Y_{n}\right)$ is the surjective map mentioned previously. 
Lemma 2.3 If $H F_{\text {red }}^{+}(Y)=0$ and $k>0$, then the group $H F^{+}\left(Y_{0}, Q^{-1}([k])\right)$ in (2) is isomorphic to ker $F$.

Proof The assumption $k>0$ implies that the map

$$
H F^{+}\left(Y_{0}, Q^{-1}([k])\right) \rightarrow H F_{r e d}^{+}\left(Y_{0}, Q^{-1}([k])\right)
$$

is an isomorphism (see Corollary 2.4 in [13]). This shows that in the commutative diagram

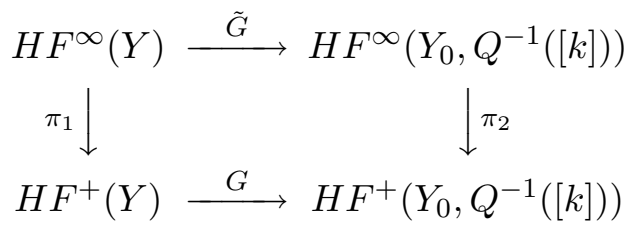

the map $\pi_{2}$ is the zero map. On the other hand, our assumption $H F_{\text {red }}^{+}(Y)=0$ shows that $\pi_{1}$ is surjective. Combined, these two observations imply that the map $G: H F^{+}(Y) \rightarrow H F^{+}\left(Y_{0}, Q^{-1}([k])\right)$ is the zero map and so the claim of the lemma follows from the exactness of (2).

Now, the map

$$
F=F_{W_{n}}: H F^{+}\left(Y_{n},[k]\right) \rightarrow H F^{+}(Y)
$$

appearing in the surgery long exact sequence is the sum of maps induced by various $\operatorname{Spin}^{c}$ structures on the cobordism $W_{n}$. Specifically, we can write $F=f_{1}+f_{2}$, where $f_{1}$ is the component of $F$ induced by the spin ${ }^{c}$-structure $\mathfrak{r}$ on $W_{n}$ extending $\mathfrak{s}$ and having $\left\langle c_{1}(\mathfrak{r}),[\tilde{F}]\right\rangle=2 k-n$, and $f_{2}$ is the sum of the maps induced by $\operatorname{spin}^{c}$-stuctures $\mathfrak{r}$ with $\left\langle c_{1}(\mathfrak{r}),[\tilde{F}]\right\rangle=2 k+(2 \ell-1) n$ with $\ell \neq 0$. Let us assume that the $\operatorname{Spin}^{c}$ structure on $Y$ (and therefore the relevant ones on $Y_{n}$ ) is torsion, so that there is an absolute grading on $H F^{+}$. This will be the case in all situations we'll consider. Then the degree shift formula (cf. [8]) for maps induced by cobordisms shows that each component of $f_{2}$ is homogeneous of degree at least $2 k$ smaller than that of $f_{1}$ (see the proof of theorem 9.1 in 9]). Using this fact, Ozsváth and Szabó prove (in section 9 of [9]) that there is a $\mathbb{Z}$-module identification $\operatorname{ker} F \cong \operatorname{ker} f_{1}$. In their case the manifold $Y$ is $\#^{2 g}\left(S^{1} \times S^{2}\right)$ but the only property of this manifold required by their proof is the fact $H F_{r e d}^{+}(Y)=0$, an assumption that we build into our discussion. On the other hand, ker $f_{1}$ can be identified as the homology of a quotient complex of $C F K^{\infty}(Y, K)$. Namely, consider the short exact sequence

$$
0 \rightarrow C\{i<0 \text { and } j \geq k\} \stackrel{\sigma}{\longrightarrow} C\{i \geq 0 \text { or } j \geq k\} \stackrel{\tau}{\longrightarrow} C\{i \geq 0\} \rightarrow 0
$$


Note that $C\{i \geq 0\}$ is simply a filtered version of $C F^{+}(Y)$. With this in mind, we have that $\operatorname{ker} f_{1}$ can be identified (as a $\mathbb{Z}$-module) with $\operatorname{ker} \tau_{*}$. This is also proved in section 9 of [9] for the case $Y=\#^{2 g}\left(S^{1} \times S^{2}\right)$ and it translates verbatim to general case provided one assumes $H F_{\text {red }}^{+}(Y)=0$. This latter assumption also implies that the connecting homomorphism $\delta: H_{*}(C\{i \geq$ $0\}) \rightarrow H_{*}(C\{i<0$ and $j \geq k\})$ is trivial and so $\operatorname{ker} \tau_{*} \cong \operatorname{Im}\left(\sigma_{*}\right) \cong H_{*}(C\{i<$ 0 and $j \geq k\})$. These remarks prove theorem 2.1

\subsection{The module structure on $H F^{+}\left(Y_{0}(K)\right)$}

Heegaard Floer homology groups come with an additional algebraic structure, namely an action of $H_{1}(M ; \mathbb{Z}) /$ Tors $\otimes_{\mathbb{Z}} \mathbb{Z}[U]$. In this section we describe this action in certain cases for $M=Y_{0}(K)$ (see theorem 2.5 below).

Observe that there are isomorphisms

$$
\begin{aligned}
& H_{1}\left(Y_{0}(K)\right) / \text { Tors } \cong\left(H_{1}(Y) / \text { Tors }\right) \oplus \mathbb{Z} \\
& H_{1}\left(Y_{n}(K)\right) / \text { Tors } \cong H_{1}(Y) / \text { Tors }
\end{aligned}
$$

In particular, $H_{1}(Y) /$ Tors acts on all three terms appearing in the long exact sequence (2). It follows from the fact that $F, G$, and $H$ in that sequence are induced by cobordisms that they are all equivariant with respect to the $H_{1}(Y) /$ Tors action (cf. [12]).

Definition 2.4 We define the maximum and minimum discrepancies $M$ and $m$ for $(Y, K, \mathfrak{t})$ to be the two integers

$$
M=\max _{x \in \widehat{H F K}(Y, K, j)} \operatorname{gr}(x)-j \quad \text { and } \quad m=\min _{x \in \widehat{H F K}(Y, K, j)} \operatorname{gr}(x)-j
$$

For a knot $K \subset Y$, we let $g$ denote the integer

$$
g=\max \{j \in \mathbb{Z} \mid \widehat{H F K}(Y, K ; j) \neq 0\} .
$$

Theorem 2.5 If $(M+g-2)-2 k<m+k$ then the isomorphism of theorem 2.1 is a $\Lambda^{*} H_{1}(Y) \otimes_{\mathbb{Z}} \mathbb{Z}[U]$ module isomorphism.

Proof This is essentially proved in the last paragraph of the proof of theorem 9.3 from $[9$. We sketch the argument here. First, it follows from the proof of ker $f_{1} \cong \operatorname{ker} \tau_{*}$ (see (3) ) that the identification in theorem 2.1] is an identification 
of $H_{1}(Y) \otimes \mathbb{Z}[U]$-modules if the map $f_{2}$ sends any element $\xi$ of ker $f_{1}$ into the group

$$
X=H_{*}(C\{i \geq 0 \text { and } j<k\}) .
$$

This can be arranged under the hypotheses of the theorem by using the degree shift formula, as follows. We impose an absolute grading on $\mathrm{HF}^{+}\left(Y_{0}, k\right)$ by using its identification with $\operatorname{ker} F$ followed by the identification of the latter as a subgroup of $H_{*}(C\{i \geq 0$ or $j \geq k\})$ via theorem [2.2. Now note that the topdegree element of ker $f_{1}$ has degree at most $M+g-2$, and compared with $f_{1}$, we know $f_{2}$ lowers degree by at least $2 k$. Furthermore, any element $\eta \in H_{*}(C\{i \geq$ $0\}$ ) having degree less than $m+k$ is necessarily contained in $X$. Hence, given $\xi \in \operatorname{ker} f_{1}$ we are assured that $f_{2}(\xi) \in X$ whenever $(M+g-2)-2 k<m+k$.

The remaining steps in the identification $\operatorname{HF}^{+}\left(Y_{0}, k\right) \cong H_{*}(C\{i \geq 0$ or $j \geq k\})$ have already been seen to be module isomorphisms.

Remark 2.6 Typically the absolute grading $g r(x)$ is a nonintegral rational number, so both $M$ and $m$ are a priori rational. However, for the purposes of applying theorem 2.5 we may replace this $\mathbb{Q}$ grading by an arbitrary $\mathbb{Z}$ grading (compatible with the relative grading), since both sides of the inequality are thereby shifted by the same amount.

The 3-manifold $Y_{0}(K)$ carries an extra generator $\mu \in H_{1}\left(Y_{0}(K)\right.$; $\left.\mathbb{Z}\right)$ coming from the meridian of $K$. The above theorem does not describe the action of this class, but we have the following:

Lemma 2.7 Suppose the homomorphism $G$ in (2) is trivial (for example, if $H F_{r e d}^{+}(Y)=0$ and $\left.k \neq 0\right)$. Then the action of $\mu$ on $H F^{+}\left(Y_{0}(K), Q^{-1}([k])\right)$ is trivial.

Proof Since $\mu$ is homologous to a torsion class in $Y_{n}$ via the cobordism connecting $Y_{0}$ to $Y_{n}$, we have

$$
H(\mu \cdot \xi)=0
$$

for $\xi \in H F^{+}\left(Y_{0}, Q^{-1}([k])\right)$. The result follows since when $G=0, H$ is injective.

\subsection{Calculational approach}

We sketch here the basis for the calculations to follow. 
Let $K \subset Y$ be a nullhomologous knot in $Y$. We will need to refer to various spin $^{c}$-structures on the triple of manifolds $Y, Y_{0}$ and $Y_{n}$. The standard 2handle cobordisms connecting these manifolds, and a choice of Seifert surface $F$ for $K$, provide identifications

$$
\operatorname{Spin}^{c}\left(Y_{0}\right) \cong \operatorname{Spin}^{c}(Y) \times \mathbb{Z} \quad \operatorname{Spin}^{c}\left(Y_{n}\right) \cong \operatorname{Spin}^{c}(Y) \times \mathbb{Z}_{n} .
$$

For example, $\tilde{\mathfrak{s}} \in \operatorname{Spin}^{c}\left(Y_{0}(K)\right)$ is identified with $(\mathfrak{s}, k) \in \operatorname{Spin}^{c}(Y) \times \mathbb{Z}$ if $\tilde{\mathfrak{s}}$ is cobordant to $\mathfrak{s}$ by a $\operatorname{Spin}^{c}$ structure $\mathfrak{r}$ on the cobordism connecting $Y$ and $Y_{0}(K)$ having $\left\langle c_{1}(\mathfrak{r}),[\hat{F}]\right\rangle=2 k$, where $\hat{F}$ denotes the Seifert surface capped off by the core of the 2-handle in the cobordism. For a $\operatorname{spin}^{c}$-structure $\mathfrak{s} \in \operatorname{Spin}^{c}(Y)$ we will write $\mathfrak{s}_{k}$ for the $\operatorname{spin}^{c}$-structure on either $Y_{0}$ or $Y_{n}$ corresponding to $(\mathfrak{s}, k)$ under the above identifications. This is consistent with the notation from the beginning of this section.

According to the results above, in order to find $H F^{+}\left(Y_{0}(K), \mathfrak{s}_{k}\right)$ we must calculate the homology of the quotient complex $C\{i<0$ and $j \geq k\}$ of $C F K^{\infty}(Y, K)$ (where the filtration on the latter group is defined using our fixed Seifert surface $F)$. To do so, we make use of a second filtration on $C F K^{\infty}$, namely

$$
\mathcal{F}^{\prime}:[\mathbf{x}, i, j] \mapsto i+j .
$$

Thinking of this as a filtration on $C F^{\infty}$, we get a spectral sequence converging to $H F^{\infty}(Y, \mathfrak{s})$. The $E^{1}$ term of this sequence may be identified with $\widehat{H F K}(Y, \mathfrak{s}) \otimes \mathbb{Z}\left[U, U^{-1}\right]$ (see lemma 3.6 in $[9]$ ).

Similarly, by restricting $\mathcal{F}^{\prime}$ to $C\{i<0$ and $j \geq k\}$ we obtain a spectral sequence whose $E^{1}$ term is

$$
Y(g, d)=\bigoplus_{i=0}^{d} \widehat{H F K}(Y, K, g-i) \otimes_{\mathbb{Z}} \mathbb{Z}[U] / U^{d+1-i} .
$$

Here we define $d=g-1-k$, and as above we let

$$
g=g_{\mathfrak{s}}=\max \{j \mid \widehat{H F K}(Y, K, \mathfrak{s}, j) \neq 0\}
$$

Our method of calculation in the following sections will be to compute the group $Y(g, d)$ and the $d_{1}$ differential in the spectral sequence. An explicit calculation of the homology follows in each case, and luckily in each case the spectral sequence collapses at the $E^{2}$ stage so that no further work is necessary.

The question remains how to find the first differential $d_{1}$ on $Y(g, d)$. From the structure of the filtration $\mathcal{F}^{\prime}$, we see that $d_{1}$ is comprised of the sum of "horizontal" and "vertical" components, the first of which decreases $i$ by one, and the second decreases $j$ by one. To understand the vertical differential, 
note that $\mathcal{F}^{\prime}$ restricts to the complex $\widehat{C F}=C\{i=0\}$ as the usual filtration induced by the knot $K$. Therefore, the corresponding $d_{1}$ differential must agree with the $d_{1}$ differential in the spectral sequence calculating $\widehat{H F}(Y, \mathfrak{s})$ from $\widehat{H F K}(Y, K, \mathfrak{s})$, which in our examples will be a simple matter to describe. The horizontal differential can be obtained similarly using the fact that $C\{j=0\}$ also is a filtered version of $\widehat{C F}$, or alternatively by formal calculation using the fact that $d_{1}^{2}=0$. More details will be given in the following sections, as need arises.

\section{Notational shorthand and a model calculation}

We begin our study of mapping tori by reviewing the calculation in 9] for the case of a single nonseparating Dehn twist. The connected sum theorem for $\widehat{H F K}$, which we frequently rely on in the remainder of the paper, can be found in section 7 of [9]. With the notation used there, it states (see 9] for more details):

$\widehat{H F K}\left(Y_{1} \# Y_{2}, K_{1} \# K_{2}, \mathfrak{t}_{3}\right) \cong \bigoplus_{\mathfrak{t}_{1}+\mathfrak{t}_{2}=\mathfrak{t}_{3}} H_{*}\left(\widehat{C F K}\left(Y_{1}, K_{1}, \mathfrak{t}_{1}\right) \otimes \widehat{C F K}\left(Y_{2}, K_{2}, \mathfrak{t}_{2}\right)\right)$

The first step is to realize this mapping torus $M\left(t_{\gamma}\right)$ as the result of 0 -surgery on some knot in a 3-manifold $Y$. Throughout, we use $\Sigma_{g}$ to denote a closed surface of genus $g$. Recall that the 3 -torus $\Sigma_{1} \times S^{1}$ can be realized as the result of 0 -surgery on each component of the Borromean rings. Alternatively, if we perform 0-surgery on two components, the remaining component describes a knot in the connected sum $S^{1} \times S^{2} \# S^{1} \times S^{2}$. Write $B(0,0)$ for this knot: then $\Sigma_{1} \times S^{1}$ is given by 0 -surgery on $B(0,0)$. To produce the mapping torus of a right-handed Dehn twist about a nonseparating curve $\gamma$ in $\Sigma_{1}$, we may modify the surgery picture by adding a -1-framed meridian to one of the surgery circles in the picture for $2\left(S^{1} \times S^{2}\right)$. Blowing down this circle gives a knot $B(1,0)$ in $S^{1} \times S^{2}$, and performing 0-surgery on $B(1,0)$ produces the desired mapping torus (see figure 1).

For general $g$, we have a surgery description of $\Sigma_{g} \times S^{1}$ as 0 -surgery on the connected sum of $g$ copies of $B(0,0)$, which is a knot in the connected sum $2 g\left(S^{1} \times S^{2}\right)$. Just as above, the mapping torus $M\left(t_{\gamma}\right)$ of a Dehn twist on $\gamma$ is obtained by 0 -surgery on $B(1,0) \#(g-1) B(0,0)$.

It is proved in [9] that the knot Floer homologies of $B(0,0), B(1,0)$, and $B(-1,0)$ (the latter corresponding to a left-handed Dehn twist) are trivial unless we use the torsion $\operatorname{Spin}^{c}$ structure on the underlying 3-manifold, and in 


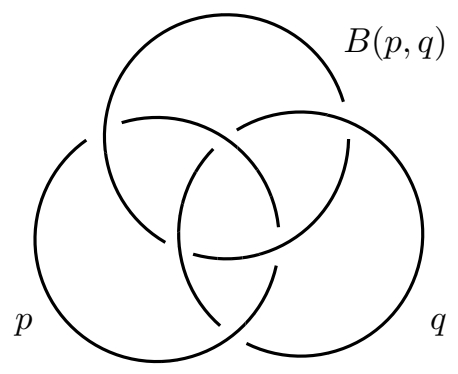

Figure 1: The knot $B(p, q)$.

this case we have:

$$
\begin{aligned}
\widehat{H F K}\left(2\left(S^{1} \times S^{2}\right), B(0,0), j\right) & \cong \Lambda^{j+1} H^{1}\left(\Sigma_{1}\right)_{(j)} \\
\widehat{H F K}\left(S^{1} \times S^{2}, B(1,0), j\right) & \cong \Lambda^{j+1} H^{1}\left(\Sigma_{1}\right)_{\left(j-\frac{1}{2}\right)} \\
\widehat{H F K}\left(S^{1} \times S^{2}, B(-1,0), j\right) & \cong \Lambda^{j+1} H^{1}\left(\Sigma_{1}\right)_{\left(j+\frac{1}{2}\right)} .
\end{aligned}
$$

The spectral sequence for $\widehat{H F}$ coming from $B(0,0)$ has no nontrivial differentials for dimensional reasons, while those coming from $B(1,0)$ and $B(-1,0)$ have nontrivial differentials only at the $E^{1}$ stage. In any case, it follows from the connected sum theorem for $\widehat{H F K}$ that as relatively graded groups all three of the knots $g B(0,0), B(1,0) \#(g-1) B(0,0)$ and $B(-1,0) \#(g-1) B(0,0)$ have knot Floer homology $\widehat{H F K}$ isomorphic (as a relatively graded group) to $\Lambda^{*} H^{1}\left(\Sigma_{g}\right)$, where the filtration on $\Lambda^{k} H^{1}\left(\Sigma_{g}\right)$ is given by $k-g$.

The $E^{1}$ term of the spectral sequence for $C\{i<0$ and $j \geq k\}$ is given in general by (5), which in this case takes the form

$$
X(g, d)=\bigoplus_{i=0}^{d} \Lambda^{2 g-i} H^{1}\left(\Sigma_{g}\right) \otimes_{\mathbb{Z}} \mathbb{Z}[U] / U^{d+1-i} .
$$

We will continue to use the notation $X(g, d)$ for the above group in all of what follows. Note that according to [4, $X(g, d)$ is isomorphic to $H_{*}\left(\operatorname{Sym}^{d}\left(\Sigma_{g}\right)\right)$, the homology of the $d$-fold symmetric product of $\Sigma_{g}$.

It is convenient to picture $X(g, d)$ as an array of groups:

$$
\begin{array}{ccccc} 
& & & & \Lambda^{2 g} H^{1} \\
& & & & \Lambda^{2 g} H^{1} \otimes U \\
& & & \Lambda^{2 g-1} H^{1} \\
\ldots & \ldots & \ldots & \vdots \\
\Lambda^{2 g} H^{1} \otimes U^{d} & \Lambda^{2 g-1} H^{1} \otimes U^{d-1} & \ldots & \ldots & \Lambda^{2 g-d} H^{1}
\end{array}
$$


Definition 3.1 We define a grading on $X(g, d)$ by the formula $\operatorname{deg}\left(\omega \otimes U^{j}\right)=$ $\operatorname{deg}(\omega)-g-2 j$, where $\operatorname{deg}(\omega)$ indicates the degree of the homogeneous element $\omega$ in the exterior algebra. We will use the notation $X(g, d)[n]$ to indicate the graded group $X(g, d)$ with the grading shifted up by $n$, and similar notation for other groups with obvious gradings.

This definition is consistent with the convention from the introduction; the grading here is the one obtained from the absolute grading on $C F K^{\infty}(g B(0,0))$. Note that this grading will not carry over to a grading on $\mathrm{HF}^{+}$for the mapping tori we are considering (the latter groups do not carry integral gradings), but it will correspond to a relative cyclic grading in those groups.

There is an action of $H_{1}\left(\Sigma_{g}\right)$ on $X(g, d)$ given as follows. Let $\gamma \in H_{1}\left(\Sigma_{g}\right)$, and define $D_{\gamma}$ by

$$
D_{\gamma}\left(\omega \otimes U^{j}\right)=\iota_{\gamma} \omega \otimes U^{j}+P D(\gamma) \wedge \omega \otimes U^{j+1} .
$$

In terms of the array of groups (8), $D_{\gamma}$ maps an element in one of the groups in the array into the two groups immediately to the left and immediately below the starting point. An easy check reveals that $D_{\gamma} D_{\gamma}=0$, thus for a fixed curve $\gamma, D_{\gamma}$ defines a differential on $X(g, d)$.

For $g \geq 2$ we write $\Sigma_{g}=\Sigma_{1} \# \Sigma_{g-1}$. This induces a splitting $\Lambda^{*} H^{1}\left(\Sigma_{g}\right)=$ $\Lambda_{+}^{*} H^{1}\left(\Sigma_{g}\right) \oplus \Lambda_{-}^{*} H^{1}\left(\Sigma_{g}\right)$ where

$$
\begin{aligned}
& \Lambda_{+}^{*} H^{1}\left(\Sigma_{g}\right)=\left(\Lambda^{0} H^{1}\left(\Sigma_{1}\right) \oplus \Lambda^{2} H^{1}(T)\right) \otimes \Lambda^{*} H^{1}\left(\Sigma_{g-1}\right) \\
& \Lambda_{-}^{*} H^{1}\left(\Sigma_{g}\right)=\Lambda^{1} H^{1}\left(\Sigma_{1}\right) \otimes \Lambda^{*} H^{1}\left(\Sigma_{g-1}\right) .
\end{aligned}
$$

This in turn induces a splitting $X(g, d)=X_{+}(g, d) \oplus X_{-}(g, d)$. Let $\gamma \subset \Sigma_{1}$ denote a fixed simple closed curve on $\Sigma_{1}$. Using the differential $D_{\gamma}$ from (9), we define two new differentials $D_{\gamma}^{ \pm}$as

$$
\left.D_{\gamma}^{ \pm}\right|_{X_{ \pm}(g, d)}=\left.0 \quad D_{\gamma}^{ \pm}\right|_{X_{\mp}(g, d)}=D_{\gamma}
$$

Remark 3.2 Our notation here differs slightly from [9], where the roles of $D_{\gamma}^{+}$ and $D_{\gamma}^{-}$are exchanged. The conventions here ensure that "+" is used for a right-handed twist throughout.

Lemma 3.3 For $K_{ \pm}$the knot $B( \pm 1,0) \#(g-1) B(0,0)$, the $d_{1}$ differential on $X(g, d)$ in the spectral sequence for $H^{+}\left(M\left(t_{\gamma}^{ \pm}\right), k\right)$ is given by $D_{\gamma}^{ \pm}$. All subsequent differentials are trivial. 
The claim about further differentials vanishing follows immediately from dimensional considerations. This lemma is proved in [9]; the fact that $d_{1}=D_{\gamma}^{ \pm}$ is a straightforward check using the description of $d_{1}$ at the end of the previous section. In particular, we can arrange the isomorphism

$$
\widehat{H F K}\left((2 g-1) S^{1} \times S^{2}, B( \pm 1,0) \#(g-1) B(0,0)\right) \cong \Lambda^{*} H^{1}\left(\Sigma_{g}\right)
$$

in such a way that the $d_{1}$ differential in the spectral sequence for $\widehat{H F}$ is trivial on $\Lambda_{+}^{*}\left(\operatorname{resp} \Lambda_{-}^{*}\right)$ and given by contraction with $\gamma$ on $\Lambda_{-}^{*}\left(\operatorname{resp} \Lambda_{+}^{*}\right)$. (This in turn is clear from the expressions (6) and the connect-sum theorem for $\widehat{H F K}$.) This shows that the vertical differential is the vertical component of $D_{\gamma}^{ \pm}$; to check the horizontal differential, one can see that it must have the claimed form by enforcing the equation $d_{1}^{2}=0$.

Our object now is to compute the homology of $\left(X(g, d), D_{\gamma}^{ \pm}\right)$. We focus on the case of a right-handed twist, meaning that the relevant differential is $D_{\gamma}^{+}$.

First we obtain an alternate description of $X_{+}(g, d)$. Let us write $c^{*} \in H^{1}\left(\Sigma_{1}\right)$ for the Poincaré dual of $\gamma$ and $c \in H^{1}\left(\Sigma_{1}\right)$ for the Poincaré dual of a geometric dual generator in homology. In other words, we have

$$
\begin{array}{ll}
\iota_{\gamma} c=1 & P D(\gamma) \wedge c=c^{*} \wedge c \\
\iota_{\gamma} c^{*}=0 & P D(\gamma) \wedge c^{*}=0 .
\end{array}
$$

Thus an element $\omega \otimes U^{j} \in X_{+}(g, d)$ ( $\omega$ a monomial) has the property that either $\omega \in \Lambda^{*} H^{1}\left(\Sigma_{g-1}\right)$ or $\omega$ is of the form $c \wedge c^{*} \wedge \lambda$ for $\lambda \in \Lambda^{*} H^{1}\left(\Sigma_{g-1}\right)$. Therefore:

$$
\begin{aligned}
X_{+}(g, d)= & \left(\bigoplus_{i=0}^{d} \Lambda^{2 g-i} H^{1}\left(\Sigma_{g-1}\right) \otimes \mathbb{Z}[U] / U^{d-i+1}\right) \\
\oplus\left(c \wedge c^{*} \wedge \bigoplus_{i=0}^{d} \Lambda^{2 g-i-2} H^{1}\left(\Sigma_{g-1}\right) \otimes \mathbb{Z}[U] / U^{d-i+1}\right) & \left(\bigoplus_{j=0}^{d-2} \Lambda^{2 g-2-j} H^{1}\left(\Sigma_{g-1}\right) \otimes \mathbb{Z}[U] / U^{(d-2)-j+1}\right) \\
= & \cong\left(c \wedge c^{*} \wedge \bigoplus_{i=0}^{d} \Lambda^{2 g-i-2} H^{1}\left(\Sigma_{g-1}\right) \otimes \mathbb{Z}[U] / U^{d-i+1}\right) \\
\cong & X(g-1, d-2)[-1] \oplus c \wedge c^{*} \wedge X(g-1, d)[-1] .
\end{aligned}
$$

(Note that in the first summand of the first line, the terms with $i=0$ and $i=1$ vanish, so the second line follows by replacing $i$ by $j=i+2$.) 
Now we consider the homology of $\left(X(g, d), D_{\gamma}^{+}\right)$. First, note that $X_{+}(g, d)$ consists of cycles by definition. To see the homology coming from $X_{+}(g, d)$, note that if $\omega \in \Lambda^{*} H^{1}\left(\Sigma_{g-1}\right)$ then from (11):

$$
\begin{aligned}
D_{\gamma}^{+}\left(c \wedge \omega \otimes U^{j}\right) & =\omega \otimes U^{j}-c \wedge c^{*} \wedge \omega \otimes U^{j+1} \\
D_{\gamma}^{+}\left(c^{*} \wedge \omega \otimes U^{j}\right) & =0
\end{aligned}
$$

From (13), it follows that the portion of the homology generated by $X_{+}(g, d)$ is simply $X_{+}(g, d)$ with the relation that $\omega \otimes U^{j}-c \wedge c^{*} \wedge \omega \otimes U^{j+1}=0$ for $\omega \in \Lambda^{*} H^{1}\left(\Sigma_{g-1}\right)$ and $j \geq 0$. In terms of the decomposition (12) of $X_{+}(g, d)$, this means that the group $X(g-1, d-2)$ appearing in (12) is identified with its image in $c \wedge c^{*} \wedge X(g-1, d)$ under the map $f\left(\omega \otimes U^{j}\right)=c \wedge c^{*} \wedge \omega \otimes U^{j+1}$. Since the latter map is injective, the homology will contain a copy of $X(g-1, d-2)$. The remainder of the homology coming from $X_{+}(g, d)$ is given by the cokernel of $f$ in $c \wedge c^{*} \wedge X(g-1, d)$, modulo any boundaries. Now we can think of $f$ as mapping one step diagonally up and to the left in the array (8) (with $g$ replaced by $g-1$ ); the cokernel of $f$ is then the "bottom row" together with the "rightmost column." An element of the rightmost column is never a boundary, while an element $c \wedge c^{*} \wedge \omega \otimes U^{j} \in X_{+}(g, d)$ that lives on the bottom row (but not in the rightmost column) is the image of $-c \wedge \omega \otimes U^{j-1}$ under $D_{\gamma}^{+}$. Thus the cokernel of $f$ contributes the group

$$
c \wedge c^{*} \wedge \bigoplus_{i=0}^{d} \Lambda^{2 g-2-i} H^{1}\left(\Sigma_{g-1}\right) \cong \bigoplus_{i=0}^{d} \Lambda^{2 g-2-i} H^{1}\left(\Sigma_{g-1}\right)_{(g-i)}
$$

to the homology. In fact we can rewrite the contribution of $X_{+}(g, d)$ in another way, as follows.

So far, $X_{+}(g, d)$ contributes

$$
X(g-1, d-2)[-1] \oplus \bigoplus_{i=0}^{d} \Lambda^{2 g-2-i} H^{1}\left(\Sigma_{g-1}\right)_{(g-i)} .
$$

Comparing this with the array (8), we see that

$$
X(g-1, d-2)[-1] \oplus \bigoplus_{i=0}^{d-1} \Lambda^{2 g-2-i} H^{1}\left(\Sigma_{g-1}\right)_{(g-i)} \cong X(g-1, d-1)[1] .
$$

Hence the contribution from $X_{+}(g, d)$ can be written as

$$
X(g-1, d-1)[1] \oplus \Lambda^{2 g-2-d} H^{1}\left(\Sigma_{g-1}\right)_{(g-d)} .
$$

Consider now the contribution from $X_{-}(g, d)$. According to (13) and (14), and the fact that no element of $X_{-}(g, d)$ can be a boundary, the homology coming 
from $X_{-}(g, d)$ is isomorphic to the group generated by elements of the form $c^{*} \wedge \omega \otimes U^{j}, \omega \in \Lambda^{*} H^{1}\left(\Sigma_{g-1}\right)$. This is

$$
\begin{aligned}
\left\langle c^{*} \wedge \omega \otimes U^{j}\right\rangle & =c^{*} \wedge \bigoplus_{i=0}^{d-1} \Lambda^{2 g-2-i} H^{1}\left(\Sigma_{g-1}\right) \otimes \mathbb{Z}[U] / U^{d-i} \\
& =c^{*} \wedge X(g-1, d-1)[-1] \cong X(g-1, d-1) .
\end{aligned}
$$

This completes the calculation of $H_{*}\left(X(g, d), D_{\gamma}^{+}\right)$; the case of $H_{*}\left(X(g, d), D_{\gamma}^{-}\right)$ is treated similarly. To summarize, we have proved the case of the following theorem relevant to a right-handed Dehn twist $t_{\gamma}$, which proves the case $n=1$ of theorem 1.1. (Note that when $n=1$ there is a unique $\operatorname{Spin}^{c}$ structure $\left.\mathfrak{s}_{k} \in \mathcal{S}_{k}.\right)$

Theorem 3.4 Let $\Sigma_{g}=\Sigma_{1} \# \Sigma_{g-1}$ be a genus $g$ surface with $g \geq 2$ and let $\gamma \subset \Sigma_{1}$ be a simple closed curve. With $X(g, d)$ and $D_{\gamma}^{ \pm}$as defined by (5D) and (10), the homology groups $H_{*}\left(X(g, d), D_{\gamma}^{ \pm}\right)$are isomorphic as relatively graded groups to $H F^{+}\left(M\left(t_{\gamma}^{ \pm 1}\right), \mathfrak{s}_{k}\right)$, where $d=g-1-|k|$. Explicitly, we have:

$$
\begin{aligned}
& H_{*}\left(X(g, d), D_{\gamma}^{+}\right)= \\
& \quad X(g-1, d-1) \oplus X(g-1, d-1)[1] \oplus \Lambda^{2 g-2-d} H^{1}\left(\Sigma_{g-1}\right)_{(g-d)} \\
& H_{*}\left(X(g, d), D_{\gamma}^{-}\right)= \\
& \quad X(g-1, d-1) \oplus X(g-1, d-1)[-1] \oplus \Lambda^{2 g-2-d} H^{1}\left(\Sigma_{g-1}\right)_{(g-d)}
\end{aligned}
$$

with $X(g-1, \cdot)[\ell]$ as in definition 3.1 .

The calculation for a left-handed twist is dual to the one just performed. While the calculation preceding theorem 3.4 focused on the case $k>0$, the case of $-k$ yields the same result. This can be seen from formula (11) together with the observation that the "background"torsion $\operatorname{spin}^{c}$-structure $\mathfrak{s}_{0}$ on $Y=$ $\#(2 g-1)\left(S^{1} \times S^{2}\right)$ is its own conjugate.

\section{Multiple Dehn twists along a non-separating curve}

In this section we calculate $H F^{+}$for the mapping torus $M\left(t_{\gamma}^{n}\right)$ where $t_{\gamma}$ denotes

the right-handed Dehn twist along a nonseparating curve $\gamma \subset \Sigma_{g}$ as usual. As in the previous section, our strategy will be to exhibit $M\left(t_{\gamma}^{n}\right)$ as the result of zero surgery along a knot and then to use the tools developed in section 2.

The mapping torus $M\left(t_{\gamma}^{n}\right)$ is obtained as zero framed surgery along the knot

$$
K=B(n, 0) \#(g-1) B(0,0)
$$


in $Y=L(n, 1) \#(2 g-1)\left(S^{1} \times S^{2}\right)$. Here, as in [9], if $p, q \in \mathbb{Q} \cup\{\infty\}$ then we can perform surgery on two components of the Borromean rings with coefficients $p$ and $q$ : the third component is the knot $B(p, q)$ (see figure 1).

We first calculate $\widehat{H F K}(Y, K)$. To do so, note that $H^{2}(Y ; \mathbb{Z}) \cong \mathbb{Z}_{n} \oplus \mathbb{Z}^{2 g-1}$, and in fact we can write a $S$ pin ${ }^{c}$ structure on $Y$ as a connected-sum $\mathfrak{t}_{i} \# \mathfrak{s}$ where $\mathfrak{s} \in \operatorname{Spin}^{c}\left((2 g-1)\left(S^{1} \times S^{2}\right)\right)$ and $\mathfrak{t}_{i} \in \operatorname{Spin}^{c}(L(n, 1)), i \in \mathbb{Z}_{n}$ is defined using the conventions from section 2 (ie, using the standard 2-handle cobordism from $S^{3}$ to $\left.L(n, 1)\right)$. We write $\mathfrak{s}_{0}$ for the torsion $\operatorname{Spin}^{c}$ structure on $\#(2 g-1) S^{1} \times S^{2}$; we also assume that $n>0$ since the case $n<0$ is treated in much the same way. First we give the calculation in the case $g=1$.

Lemma 4.1 The knot Floer homology $\widehat{H F K}\left(L(n, 1) \#\left(S^{1} \times S^{2}\right), B(n, 0), \mathfrak{t}_{i} \# \mathfrak{s}\right)$ is zero unless $\mathfrak{s}=\mathfrak{s}_{0}$. In this case, if $i \neq 0$ we have isomorphisms of relatively graded groups

$$
\widehat{H F K}\left(L(n, 1) \#\left(S^{1} \times S^{2}\right), B(n, 0), \mathfrak{t}_{i} \# \mathfrak{s}_{0}, j\right) \cong\left\{\begin{array}{cl}
H^{*}\left(S^{1} ; \mathbb{Z}\right) & j=0 \\
0 & \text { otherwise }
\end{array}\right.
$$

while for $i=0$ we have

$$
\widehat{H F K}\left(L(n, 1) \#\left(S^{1} \times S^{2}\right), B(n, 0), \mathfrak{t}_{0} \# \mathfrak{s}_{0}, j\right) \cong \Lambda^{j+1} H^{1}\left(\Sigma_{1} ; \mathbb{Z}\right),
$$

where $\Sigma_{1}$ denotes a genus-1 surface. The spectral sequence for $\widehat{C F}$ induced by $K$ collapses at the $E^{1}$ level for $i \neq 0$ and collapses at the $E^{2}$ level for $i=0$. In the latter case, the $d_{1}$ differential is trivial except for its restriction to the $j=0$ summand

$$
d_{1}: \Lambda^{1} H^{1}\left(\Sigma_{1}\right) \rightarrow \Lambda^{0} H^{1}\left(\Sigma_{1}\right)
$$

which is surjective.

Proof The starting point for this calculation is the surgery long exact sequence in knot Floer homology (see [10] and [9]):

$$
\begin{aligned}
\cdots \rightarrow \widehat{H F K}\left(S^{1} \times S^{2}, B(\infty, 0), \mathfrak{s}, j\right) \rightarrow \widehat{H F K}\left(\#^{2}\left(S^{1} \times S^{2}\right), B(0,0),\left[\mathfrak{s}_{i}\right] \# \mathfrak{s}, j\right) \rightarrow \\
\rightarrow \widehat{H F K}\left(L(n, 1) \#\left(S^{1} \times S^{2}\right), B(n, 0), \mathfrak{t}_{i} \# \mathfrak{s}, j\right) \rightarrow \cdots
\end{aligned}
$$

Of course $B(\infty, 0)$ is just the unknot in $S^{1} \times S^{2}$. When $\mathfrak{s} \neq \mathfrak{s}_{0}$, both the first and second term in (18) vanish by the connected sum theorem for $\widehat{H F K}$, so the first claim of the lemma is immediate. For $\mathfrak{s}=\mathfrak{s}_{0}$, there are several cases to consider, depending on $\mathfrak{t}_{i}$ as well as the filtration index $j$. 
When $i \neq 0$ then the second term in (18) is zero and the first term is only nonzero for $j=0$. Indeed, since the unknot induces the trivial filtration on $\widehat{C F}$, the knot Floer homology of the unknot in $Y$ is isomorphic to $\widehat{H F}(Y)$ supported in filtration level zero. Since $\widehat{H F}\left(S^{1} \times S^{2} ; \mathfrak{s}_{0}\right) \cong H^{*}\left(S^{1} ; \mathbb{Z}\right)$ as relatively graded groups, (16) follows.

If $i=0$ and $j \neq 0$, then the first term in (18) is zero and the second term is nonzero only if $j= \pm 1$ (cf. equation (6) ). Since $\widehat{H F K}(B(0,0), j) \cong \mathbb{Z}$ when $j= \pm 1$, the statement follows in this case.

In the remaining case $i=j=0$, (18) easily shows that $\widehat{H F K}(B(n, 0))$ can be nonzero only in two adjacent degrees: let us call them $a$ and $b$. Then the sequence becomes

$$
\begin{aligned}
0 \rightarrow \widehat{H F K}_{a}(L & \left.(n, 1) \#\left(S^{1} \times S^{2}\right), B(n, 0), \mathfrak{t}_{0} \# \mathfrak{s}_{0}, 0\right) \rightarrow \mathbb{Z}_{\left(\frac{1}{2}\right)} \rightarrow \mathbb{Z}_{(0)}^{2} \rightarrow \\
& \rightarrow \widehat{H F K}_{b}\left(L(n, 1) \#\left(S^{1} \times S^{2}\right), B(n, 0), \mathfrak{t}_{0} \# \mathfrak{s}_{0}, 0\right) \rightarrow \mathbb{Z}_{\left(-\frac{1}{2}\right)} \rightarrow 0
\end{aligned}
$$

and it is an exercise to see that the map $\mathbb{Z}_{\left(\frac{1}{2}\right)} \rightarrow \mathbb{Z}_{(0)}^{2}$ is injective. This completes the proof, as the statements about the spectral sequence follow easily from dimensional considerations.

For the case of general $g$ in (15), the connected sum theorem for $\widehat{H F K}$ combined with the previous lemma yields:

Lemma 4.2 The Heegaard knot Floer homology $\widehat{H F K}(Y, K, \mathfrak{t})$ is zero unless $\mathfrak{t}=\mathfrak{t}_{i} \#^{2 g-1} \mathfrak{s}_{0}$ for some $i$ and in this case is given by

$$
\widehat{H F K}\left(Y, K, \mathfrak{t}_{i} \#^{2 g-1} \mathfrak{s}_{0}\right) \cong \begin{cases}\Lambda^{*}\left(H^{1}\left(S^{1} ; \mathbb{Z}\right) \oplus H^{1}\left(\Sigma_{g-1} ; \mathbb{Z}\right)\right) & i \neq 0 \\ \Lambda^{*} H^{1}\left(\Sigma_{g} ; \mathbb{Z}\right) & i=0\end{cases}
$$

The spectral sequence for $\widehat{C F}$ induced by $\widehat{C F K}$ collapses at the $E^{1}$ level for $i \neq 0$ and it collapses at the $E^{2}$ level for $i=0$. The $d_{1}$ differential in the latter case is trivial except on $H^{1}\left(\Sigma_{1}\right)$ where it is contraction with $\gamma$ (a generator of $\left.H^{1}\left(\Sigma_{1}\right)\right)$.

Let $Y(g, d)$ be as in (5). In the case $i=0$ we see from (19) that in fact $Y(g, d)=X(g, d)$. Thus the splitting $\Sigma_{g}=\Sigma_{1} \# \Sigma_{g-1}$ induces the decomposition $Y(g, d)=X(g, d)=X_{+}(g, d) \oplus X_{-}(g, d)$ as in section 3 the $d_{1}$ differential from lemma 4.2 gives rise to a differential $D$ on $Y(g, d)$ which can easily be 
seen to be given by $D_{\gamma}^{+}$. Just as in the case $n=1$ there can be no differentials beyond $d_{1}$ in the spectral sequence for $H_{*}(C\{i<0$ and $j \geq k\})$, so according to theorem 2.1 the Floer homology $\mathrm{HF}^{+}\left(M\left(t_{\gamma}^{n}\right)\right)$ is given as the homology $H_{*}(Y(g, d), D)$ (with $D=0$ if $i \neq 0$ ). The assumptions $H F_{r e d}^{+}(Y)=$ $H F_{\text {red }}^{+}\left(Y_{n}\right)=0$ of theorem 2.1 are readily verified. For the case $i \neq 0$, lemma 4.2 shows that there can be no $d_{1}$ differential in the spectral sequence for $\widehat{H F}$ indeed since the right hand side of (19) is equal to $\widehat{H F}\left(Y, t_{i}\right)$ the spectral sequence collapses at the outset.

For stating the main theorem of this section, we will use the notation $\mathfrak{t}_{i, k}$ to denote the $\operatorname{spin}^{c}$-structure $\left(\mathfrak{t}_{i} \# \mathfrak{s}_{0}, k\right) \in \operatorname{Spin}^{c}(Y) \times \mathbb{Z} \cong \operatorname{Spin}^{c}\left(M\left(t_{\gamma}^{n}\right)\right)$.

Theorem 4.3 Let $M\left(t_{\gamma}^{n}\right)$ be the mapping torus of $n$ right-handed Dehn twists along a non-separating curve $\gamma \subset \Sigma_{g}, g \geq 2$, where $n \in \mathbb{Z} \backslash\{0\}$. Pick an integer $k$ with $0<|k| \leq g-1$. Then the Heegaard Floer homology of $M\left(t_{\gamma}^{n}\right)$ for the $\operatorname{spin}^{c}$-structure $\mathfrak{t}_{i, k}$ is given as a relatively graded group by

$$
H F^{+}\left(M\left(t_{\gamma}^{n}\right), \mathfrak{t}_{i, k}\right) \cong \begin{cases}H^{*}\left(S^{1}\right) \otimes X(g-1, d-1) & i \neq 0 \\ H_{*}\left(X(g, d), D_{\gamma}^{s(n)}\right) & i=0\end{cases}
$$

where $s(n)=\operatorname{sign}(n)$ and $d=g-1-|k|$. If in addition $g<3 k+2$, the above isomorphisms are $\Lambda^{*} H_{1}(Y) \otimes_{\mathbb{Z}} \mathbb{Z}[U]$ module isomorphisms.

The claims about the module structure follow easily from lemma 4.2 and theorem 2.5. The groups $H_{*}\left(X(g, d), D_{\gamma}^{ \pm}\right)$have explicitly been calculated in theorem 3.4 and are given by

$$
\begin{aligned}
& H_{*}\left(X(g, d), D_{\gamma}^{+}\right)= \\
& \quad X(g-1, d-1) \oplus X(g-1, d-1)[1] \oplus \Lambda^{2 g-2-d} H^{1}\left(\Sigma_{g-1}\right)_{(g-d)} \\
& H_{*}\left(X(g, d), D_{\gamma}^{-}\right)= \\
& \quad X(g-1, d-1) \oplus X(g-1, d-1)[-1] \oplus \Lambda^{2 g-2-d} H^{1}\left(\Sigma_{g-1}\right)_{(g-d)}
\end{aligned}
$$

This proves theorem 1.1 for $k>0$. The case of $k<0$ follows from the observation that $\mathfrak{t}_{i,-k}=\overline{\mathfrak{t}_{n-i, k}}$. In particular, $\mathfrak{t}_{0,-k}=\overline{\mathfrak{t}_{0, k}}$. Since $H F^{+}\left(M\left(t_{\gamma}^{n}\right), \mathfrak{t}_{i, k}\right)$ (in the case when $k>0$ ) only depends on if $i=0$ or $i \neq 0$ (a property which is preserved under conjugation of the $\operatorname{spin}^{c}$-structure ), the theorem for $k<0$ now readily follows from formula (11). 


\section{Multiple Dehn twists along a transverse pair of curves}

Let $\gamma, \delta \subset \Sigma_{1}$ be a pair of curves representing generators of $H_{1}\left(\Sigma_{1}\right)$ with $\gamma \cap \delta$ being a single point. Write $\Sigma_{g}=\Sigma_{1} \# \Sigma_{g-1}$ (with $g \geq 2$ ) and think of $\gamma$ and $\delta$ as curves in $\Sigma_{g}$. We consider the mapping torus $M\left(t_{\gamma}^{m} t_{\delta}^{n}\right)$.

The manifold $M\left(t_{\gamma}^{m} t_{\delta}^{n}\right)$ can be described as the result of zero surgery along the knot

$$
K=B(m, n) \#(g-1) B(0,0)
$$

inside $Y=L(m, 1) \# L(n, 1) \#(2 g-2)\left(S^{1} \times S^{2}\right)$. Using the standard 2-handle cobordism from $(2 g-2)\left(S^{1} \times S^{2}\right)$ to $Y$ (having two handles), we have an identification as in section 2

$$
\operatorname{Spin}^{c}(Y) \cong \mathbb{Z}_{m} \oplus \mathbb{Z}_{n} \oplus \operatorname{Spin}^{c}\left((2 g-2)\left(S^{1} \times S^{2}\right)\right) .
$$

We will use $\mathfrak{t}_{a, b}$ to denote the $\operatorname{spin}^{c}$-structure corresponding to $\left(a, b, \mathfrak{s}_{0}\right)$ under this identification. To calculate the knot Floer homology of $K$, we first calculate the knot Floer homology of $B(m, n)$ and then use the connected sum theorem for $\widehat{H F K}$. The discussion proceeds by considering the two cases $m \cdot n>0$ and $m \cdot n<0$ separately.

\subsection{The case $m \cdot n>0$}

We assume that $m$ and $n$ are both positive for the moment. We find the knot Floer homology of $B(m, n)$ from the surgery long exact sequence:

$$
\begin{aligned}
\cdots \rightarrow \widehat{H F K}\left(L(m, 1) \# S^{3}, B(m, \infty), \mathfrak{t}_{a}\right) \rightarrow \\
\rightarrow \widehat{H F K}\left(L(m, 1) \#\left(S^{1} \times S^{2}\right), B(m, 0),\left[\mathfrak{t}_{a, b}\right]\right) \rightarrow \\
\quad \rightarrow \widehat{H F K}\left(L(m, 1) \# L(n, 1), B(m, n), \mathfrak{t}_{a, b}\right) \rightarrow \cdots
\end{aligned}
$$

In the above, $B(m, \infty)$ is the unknot in $L(m, 1)$ and $\widehat{H F K}\left(L(m, 1) \#\left(S^{1} \times\right.\right.$ $\left.\left.S^{2}\right), B(m, 0), \mathfrak{t}_{a, b}\right)$ was calculated in lemma 4.1. From this one can see that the sequence (21) essentially takes two possible forms depending on if $b=0$ or if $b \neq 0$. In the case when $b=0$ there is a further distinction between the cases $a=0$ and $a \neq 0$. What follows is a case by case analysis of the sequence (21). 
Recall (see [8]) that $\widehat{H F}\left(L(m, 1), \mathfrak{t}_{a}\right) \cong \mathbb{Z}$ for all $a \in\{0, \ldots, m-1\}$, and is supported in degree $\ell_{m, a}$ where

$$
\ell_{m, a}=\frac{1}{4}\left(\frac{(2 a-m)^{2}}{m}-1\right)
$$

\subsubsection{The case $b \neq 0$.}

In this case the middle term of (21) is zero while the first term is nonzero only when $j=0$ :

$$
0 \rightarrow \widehat{H F K}\left(L(m, 1) \# L(n, 1), B(m, n), \mathfrak{t}_{a, b}, 0\right) \rightarrow \mathbb{Z}_{\left(\ell_{m, a}\right)} \rightarrow 0
$$

\subsubsection{The case $b=0$ and $a \neq 0$.}

The long exact sequence (21) in this case becomes (for $j=0$ )

$$
\begin{aligned}
& 0 \rightarrow \mathbb{Z}_{\left(\ell_{m, a}+\frac{1}{2}\right)} \rightarrow \widehat{H F K}_{\left(\ell_{m, a}+\ell_{n, 0}\right)}\left(L(m, 1) \# L(n, 1), B(m, n), \mathfrak{t}_{a, 0}, 0\right) \rightarrow \\
& \mathbb{Z}_{\left(\ell_{m, a}\right)} \rightarrow \mathbb{Z}_{\left(\ell_{m, a}-\frac{1}{2}\right)} \rightarrow \widehat{H F K}_{\left(\ell_{m, a}+\ell_{n, 0}-1\right)}\left(L(m, 1) \# L(n, 1), B(m, n), \mathfrak{t}_{a, 0}, 0\right) \rightarrow 0
\end{aligned}
$$

It is easy to check that $\mathbb{Z}_{\left(\ell_{m, a}\right)} \rightarrow \mathbb{Z}_{\left(\ell_{m, a}-\frac{1}{2}\right)}$ is an isomorphism.

\subsubsection{The case $b=0$ and $a=0$.}

In this case the sequence (21) splits into three exact sequences corresponding to $j=-1,0,1$. In the cases $j= \pm 1$ the first term in (21) is zero which reduces the sequence to

$$
\begin{aligned}
0 \rightarrow \widehat{H F K}(L(m, 1) & \left.\#\left(S^{1} \times S^{2}\right), B(n, 0),\left[\mathfrak{t}_{0,0}\right], j\right) \rightarrow \\
& \rightarrow \widehat{H F K}\left(L(m, 1) \# L(n, 1), B(m, n), \mathfrak{t}_{0,0}, j\right) \rightarrow 0
\end{aligned}
$$

On the other hand, when $j=0$ the sequence (21) becomes

$$
\begin{aligned}
0 & \rightarrow \widehat{H F K}_{\left(\ell_{m, 0}+\ell_{n, 0}\right)}\left(L(m, 1) \# L(n, 1), B(m, n), \mathfrak{t}_{0,0}, 0\right) \rightarrow \mathbb{Z}_{\left(\ell_{m, 0}\right)} \rightarrow \\
& \rightarrow \mathbb{Z}_{\left(\ell_{m, 0}-\frac{1}{2}\right)}^{2} \rightarrow \widehat{H F K}_{\left(\ell_{m, 0}+\ell_{n, 0}-1\right)}\left(L(m, 1) \# L(n, 1), B(m, n), \mathfrak{t}_{0,0}, 0\right) \rightarrow 0,
\end{aligned}
$$

and one checks that $\mathbb{Z}_{\left(\ell_{m, 0}\right)} \rightarrow \mathbb{Z}_{\left(\ell_{m, 0}-\frac{1}{2}\right)}^{2}$ is injective. We summarize the above analysis in the following lemma: 
Lemma 5.1 Suppose $a$ and $b$ are not both zero. Then the knot Floer homology of $B(m, n)$ in $L(m, 1) \# L(n, 1)$ with $m, n>0$ in the $\operatorname{spin}^{c}$-structure $\mathfrak{t}_{a, b}$ is given by

$$
\widehat{H F K}\left(L(m, 1) \# L(n, 1), B(m, n), \mathfrak{t}_{a, b}\right) \cong \mathbb{Z},
$$

supported in filtration level zero and degree $\ell_{m, a}+\ell_{n, b}$.

If $a=b=0$, then

$$
\widehat{H F K}\left(L(m, 1) \# L(n, 1), B(m, n), \mathfrak{t}_{0,0}, j\right) \cong \begin{cases}\mathbb{Z}_{\left(\ell_{m, 0}+\ell_{n, 0}\right)} & j=1 \\ \mathbb{Z}_{\left(\ell_{m, 0}+\ell_{n, 0}-1\right)} & j=0 \\ \mathbb{Z}_{\left(\ell_{m, 0}+\ell_{n, 0}-2\right)} & j=-1 \\ 0 & \text { otherwise, }\end{cases}
$$

where $\ell_{m, a}$ and $\ell_{n, b}$ are given by (22). The spectral sequence on $\widehat{C F}$ induced by $\widehat{C F K}$ collapses at the $E^{1}$ level if either $a \neq 0$ or $b \neq 0$ and it collapses at the $E^{2}$ level if $a=b=0$. In the latter case, the $d_{1}$ differential is the surjective map from $\mathbb{Z}_{\left(\ell_{m, 0}+\ell_{n, 0}-1\right)} \rightarrow \mathbb{Z}_{\left(\ell_{m, 0}+\ell_{n, 0}-2\right)}$.

At the beginning of this section we assumed $m, n>0$. The knot Floer groups of $B(m, n)$ for $m, n<0$ can be found from the symmetry relation

$$
\widehat{H F K}_{d}\left(B(m, n), \mathfrak{t}_{a, b}, j\right)=\widehat{H F K}_{-d}\left(B(-m,-n), \mathfrak{t}_{a, b},-j\right)
$$

Our calculation for the knot Floer homology of $B(m, n)$ immediately yields the knot Floer homology of the knot $K$ from (20). To express the result in a notation-friendly way, we'll denote by $\Sigma_{1 / 2}$ the (fictitious) surface of genus $1 / 2$ whose cohomology is given by $H^{k}\left(\Sigma_{1 / 2} ; \mathbb{Z}\right)=\mathbb{Z}$ for $k=0,1,2$.

Lemma 5.2 For $m, n>0$ the knot Floer homology of $K$ with respect to the spin $^{c}$-structure $\mathfrak{t}_{a, b}$ is

$$
\widehat{H F K}\left(Y, K, \mathfrak{t}_{a, b}\right)= \begin{cases}\Lambda^{*} H^{1}\left(\Sigma_{g-1}\right) & a \neq 0 \text { or } b \neq 0 \\ H^{*}\left(\Sigma_{1 / 2}\right) \otimes \Lambda^{*} H^{1}\left(\Sigma_{g-1}\right) & a=b=0\end{cases}
$$

with an appropriate shift in grading on the right-hand side. In both cases the filtration is equal to the grading modulo a shift. When $a \neq 0$ or $b \neq 0$ the spectral sequence on $\widehat{C F}$ induced by $\widehat{C F K}$ collapses at the $E^{1}$ level and it collapses at the $E^{2}$ level when $a=b=0$.

Let $Y(g, d)$ the usual groups (see section 3 ) defined from $\widehat{H F K}\left(Y, K, \mathfrak{t}_{a, b}\right)$. In the case $a=b=0$, the $d_{1}$ differential from lemma 5.2 induces a differential $D$ on $Y(g, d)$, while if $a \neq 0$ or $b \neq 0$ all differentials in the spectral sequence 
starting with $Y(g, d)$ are trivial since in that case $H F K^{\infty} \otimes \mathbb{Z}\left[U, U^{-1}\right]$ is isomorphic to $H F^{\infty}$ of the underlying manifold. To give an explicit formula for $D$, we decompose $H^{*}\left(\Sigma_{1 / 2}\right)$ as $H^{+}\left(\Sigma_{1 / 2}\right) \oplus H^{-}\left(\Sigma_{1 / 2}\right)$ with

$$
\begin{aligned}
& H^{+}\left(\Sigma_{1 / 2}\right)=H^{0}\left(\Sigma_{1 / 2}\right) \oplus H^{2}\left(\Sigma_{1 / 2}\right) \\
& H^{-}\left(\Sigma_{1 / 2}\right)=H^{1}\left(\Sigma_{1 / 2}\right)
\end{aligned}
$$

This induces a corresponding decomposition of $Y(g, k)=Y_{+}(g, k) \oplus Y_{-}(g, k)$. One can then check that the differential $D$ becomes

$$
\left.D\right|_{Y_{+}(g, k)}=0 \quad D\left(c \otimes \omega \otimes U^{i}\right)=\omega \otimes U^{i}+S \wedge \omega \otimes U^{i+1}
$$

when $m, n>0$ and it is

$$
\left.D\right|_{Y_{-}(g, k)}=0 \quad D\left(\omega \otimes U^{i}\right)=c \otimes \omega \otimes U^{i+1} \quad D\left(S \otimes \omega \otimes U^{i}\right)=c \wedge \omega \otimes U^{i}
$$

when $m, n<0$. As in the case of a single twist, there can be no further differentials in the spectral sequence for $H_{*}(C\{i<0$ and $j \geq k\})$. Assembling all the above we arrive at:

Theorem 5.3 Let $M\left(t_{\gamma}^{m} t_{\delta}^{n}\right)$ be the mapping torus associated to the diffeomorphism given by $n$ Dehn twists along $\delta$ followed by $m$ Dehn twists along $\gamma$. We assume that $m \cdot n>0$ and that $\gamma, \delta \subset \Sigma_{g}$ are geometrically dual curves supported in a genus 1 summand of $\Sigma_{g}$. Then for $k \neq 0$ the Heegaard Floer homology of $M\left(t_{\gamma}^{m} t_{\delta}^{n}\right)$ is given by

$$
H F^{+}\left(M\left(t_{\gamma}^{m} t_{\delta}^{n}\right), \mathfrak{t}_{a, b, k}\right)= \begin{cases}X(g-1, d-1) & a \neq 0 \text { or } b \neq 0 \\ H_{*}(Y(g, d), D) & a=b=0\end{cases}
$$

with $D$ given by (23) when $m, n>0$ or (24) when $m, n<0$, and where $d=g-1-|k|$. If in addition $g<3 k+2$, the above isomorphisms are $\Lambda^{*} H_{1}(Y) \otimes_{\mathbb{Z}} \mathbb{Z}[U]$ module isomorphisms.

The homology groups $H_{*}(Y(g, d), D)$ can be calculated explicitly using calculations similar to those in section 3. We focus on the case $m, n>0$ first. Notice that if we let $c$ denote a generator of $H^{1}\left(\Sigma_{1 / 2}\right)$ and $S$ a generator of $H^{2}\left(\Sigma_{1 / 2}\right)$, then one can rewrite the groups $Y_{ \pm}(g, d)$ as

$$
\begin{aligned}
& Y_{+}(g, d)=X(g-1, d-2) \oplus(S \otimes X(g-1, d)) \\
& Y_{-}(g, d)=c \otimes X(g-1, d-1)
\end{aligned}
$$

From this it is easy to see that $Y_{-}(g, d)$ has no cycles while the boundaries in $Y_{+}(g, d)$ are of the form $\omega \otimes U^{i}+S \otimes \omega \otimes U^{i+1}$ for $\omega \otimes U^{i} \in X(g-1, d-2)$. The homology we seek is then

$$
Y_{+}(g, d) /\left\{\omega \otimes U^{i}+S \otimes \omega \otimes U^{i+1} \mid \omega \otimes U^{i} \in X(g-1, d-2)\right\}
$$


The above can easily be seen using arguments as in section 3 to be isomorphic to $X(g-1, d-1) \oplus \Lambda^{2 g-d-2} H^{1}\left(\Sigma_{g-1}\right)$.

In the case when $m, n<0$, all elements of $Y_{-}(g, d)$ are boundaries and don't contribute to the homology. The cycles in $Y_{+}(g, d)$ are the elements of the form $\omega \otimes U^{i}-S \otimes \omega \otimes U^{i+1}$ (contributing an $X(g-1, d-2)$ summand to the homology) as well as elements in the "bottom-most row"in $Y_{+}(g, d)$, that is elements of the form $\omega \otimes U^{j-k-1}$ with $\omega \in \Lambda^{g+j} H^{1}\left(\Sigma_{g-1}\right), j=k, \ldots, g-1$. It is an explicit check to see that the $X(g-1, d-2)$ summand together with the elements of the form $\omega \otimes U^{j-k-1}, \omega \in \Lambda^{g+j} H^{1}\left(\Sigma_{g-1}\right), j=k+1, \ldots, g-1$ add up to $X(g-1, d-1)$. A little extra care with gradings leads to

Corollary 5.4 With the assumption as in theorem 5.3 the Floer homology of $M\left(t_{\gamma}^{m} t_{\delta}^{n}\right)$ for $m \cdot n>0$ in the spin $^{c}$-structures $\mathfrak{t}_{0,0, k}$ (with $k \neq 0$ ) is

$$
H F^{+}\left(M\left(t_{\gamma}^{m} t_{\delta}^{n}\right), \mathfrak{t}_{0,0, k}\right)=\left\{\begin{array}{l}
X(g-1, d-1) \oplus \Lambda^{2 g-d-2} H^{1}\left(\Sigma_{g-1}\right)_{(g-1-d)} \\
X(g-1, d-1)[-2] \oplus \Lambda^{2 g-d-2} H^{1}\left(\Sigma_{g-1}\right)_{(g-1-d)}
\end{array}\right.
$$

The first line on the right-hand side above corresponds to the case $m, n>0$ while the second line describes the case $m, n<0$.

\section{$5.2 \quad$ The case $m \cdot n<0$}

We content ourselves by only pointing out the major differences that occur here compared with the previous case.

We may assume that $n>0$. Then the long exact sequence (21) still applies with the values of the first two terms appropriately adjusted. An easy case by case analysis (depending on $a$ and $b$ ) leads to:

Lemma 5.5 Suppose $a$ and $b$ are not both zero. Then the knot Floer homology of $B(m, n)$ with $m \cdot n<0$ in the spin ${ }^{c}$-structure $\mathfrak{t}_{a, b}$ is given by

$$
\widehat{H F K}\left(L(m, 1) \# L(n, 1), B(m, n), \mathfrak{t}_{a, b}\right) \cong \mathbb{Z},
$$

supported in filtration level zero and degree $\ell_{m, a}+\ell_{n, b}$.

If $a=b=0$, then

$$
\widehat{H F K}\left(L(m, 1) \# L(n, 1), B(m, n), \mathfrak{t}_{0,0}, j\right) \cong \begin{cases}\mathbb{Z}_{\left(\ell_{n, 0}-\ell_{m, 0}+1\right)} & j=1 \\ \mathbb{Z}_{\left(\ell_{n, 0}-\ell_{m, 0}\right)}^{3} & j=0 \\ \mathbb{Z}_{\left(\ell_{n, 0}-\ell_{m, 0}-1\right)} & j=-1 \\ 0 & \text { otherwise }\end{cases}
$$


where $\ell_{m, a}$ and $\ell_{n, b}$ are defined by (22). The induced spectral sequence on $\widehat{C F}$ collapses at the $E^{1}$ stage if either $a \neq 0$ or $b \neq 0$ and it collapses at the $E^{2}$ stage when $a=b=0$.

When $m \cdot n<0$, the symmetries of $\widehat{H F K}$ show that the knot Floer homologies of $B(m, n)$ and $B(-m,-n)$ are equal.

In the following, we will drop the reference to the absolute grading given above since only the relative grading is relevant in the end result. However, it will be convenient to suppose that the group $\widehat{H F K}(B(m, n), j)$ is supported in degree $j$ since with this convention the gradings on $Y(g, d)$ and $X(g, d)$ are compatible in the calculations to follow.

In analogy with the previous section, we introduce the genus $3 / 2$ surface $\Sigma_{3 / 2}$ whose cohomology groups are $H^{k}\left(\Sigma_{3 / 2} ; \mathbb{Z}\right)=\mathbb{Z}$ for $k=0,2$ and $H^{1}\left(\Sigma_{3 / 2}\right)=\mathbb{Z}^{3}$.

Lemma 5.6 For $m \cdot n<0$ the knot Floer homology of $K$ (given by (20)) with respect to the $\operatorname{spin}^{c}$-structure $\mathfrak{t}_{a, b}$ is

$$
\widehat{H F K}\left(Y, K, \mathfrak{t}_{a, b}\right)= \begin{cases}\Lambda^{*} H^{1}\left(\Sigma_{g-1}\right) & a \neq 0 \text { or } b \neq 0 \\ H^{*}\left(\Sigma_{3 / 2}\right) \otimes \Lambda^{*} H^{1}\left(\Sigma_{g-1}\right) & a=b=0\end{cases}
$$

with an appropriate shift in grading on the right-hand side. In both cases the filtration is equal to the grading modulo a shift. When $a \neq 0$ or $b \neq 0$ the spectral sequence on $\widehat{C F}$ induced by $\widehat{C F K}$ collapses after the $E^{1}$ level and it collapses after the $E^{2}$ level when $a=b=0$.

When $a=b=0$, the $d_{1}$ differential in $\widehat{H F K}$ can be described as follows. Since the underlying manifold of $B(m, n)$ has $\widehat{H F} \cong \mathbb{Z}$ and the filtration in $\widehat{H F K}$ is equal to the grading, the $d_{1}$ differential must correspond to a differential on $H^{*}\left(\Sigma_{3 / 2}\right)$ whose homology is $\mathbb{Z}$ supported in the middle dimension. Let $e$ be a generator for $H^{0}\left(\Sigma_{3 / 2}\right)$, write $c_{1}, c_{2}, c_{3}$ for the generators of $H^{1}\left(\Sigma_{3 / 2}\right)$, and write a generator of $H^{2}\left(\Sigma_{3 / 2}\right)$ as $S$. Then under the $d_{1}$ differential we can suppose that $S \mapsto c_{1}$ and that $c_{2} \mapsto e$ and $c_{3} \mapsto e$. From this it is easy to check that for $\omega \in \Lambda^{*} H^{1}\left(\Sigma_{g-1}\right)$, the differential $D$ on $Y(g, d)$ must be given by:

$$
\begin{aligned}
& D\left(e \otimes \omega \otimes U^{i}\right)=c_{1} \otimes \omega \otimes U^{i+1} \\
& D\left(c_{\ell} \otimes \omega \otimes U^{i}\right)= \begin{cases}0 & \ell=1 \\
e \otimes \omega \otimes U^{i}-S \otimes \omega \otimes U^{i+1} & \ell=2,3\end{cases} \\
& D\left(S \otimes \omega \otimes U^{i}\right)=c_{1} \otimes \omega \otimes U^{i}
\end{aligned}
$$

There are no further differentials in the spectral sequence for dimensional reasons, so we get: 
Theorem 5.7 Let $M\left(t_{\gamma}^{m} t_{\delta}^{n}\right)$ be the mapping torus associated to the diffeomorphism given by $n$ right-handed Dehn twists along $\delta$ followed by $m$ right-handed Dehn twists along $\gamma$. Assume that $m \cdot n<0$ and that $\gamma, \delta \subset \Sigma_{g}$ are geometrically dual curves supported in a genus 1 summand of $\Sigma_{g}$. Then the Heegaard Floer homology of $M\left(t_{\gamma}^{m} t_{\delta}^{n}\right)$ for $k \neq 0$ is given by

$$
H F^{+}\left(M\left(t_{\gamma}^{m} t_{\delta}^{n}\right), \mathfrak{t}_{a, b, k}\right)= \begin{cases}X(g-1, d-1) & a \neq 0 \text { or } b \neq 0 \\ H_{*}(Y(g, d), D) & a=b=0\end{cases}
$$

with $D$ given by (25). If in addition $g<3 k+2$, the above isomorphisms are $\Lambda^{*} H_{1}(Y) \otimes_{\mathbb{Z}} \mathbb{Z}[U]$ module isomorphisms.

As in the previous section, the group $H_{*}(Y(g, d), D)$ can be calculated explicitly. Once again, our first step is to rewrite $Y(g, d)$ appropriately. We write

$$
H^{*}\left(\Sigma_{3 / 2}\right)=A_{1} \oplus A_{0} \oplus A_{-1},
$$

where $A_{1}=\operatorname{span}\left\{c_{2}, c_{3}\right\}, A_{0}=\operatorname{span}\{S, e\}$, and $A_{-1}=\operatorname{span}\left\{c_{1}\right\}$. This induces a corresponding splitting of $\widehat{H F K}(Y, K)$, and thence a decomposition

$$
Y(g, d)=X_{1} \oplus X_{0} \oplus X_{-1} .
$$

From (25), we see that $D$ maps $X_{i}$ into $X_{i-1}$ (and vanishes on $X_{-1}$ ). Clearly $D$ maps onto $X_{-1}$.

The group of cycles in $X_{0}$ is generated by elements of the form $\xi=e \otimes \omega \otimes U^{i-1}$ $S \otimes \omega \otimes U^{i}$, where $\omega \in \Lambda^{j} H^{1}\left(\Sigma_{g-1}\right)$. Such an element is the boundary of $c_{2} \otimes \omega \otimes$ $U^{i-1}$ except in the case $i=0$. This is the situation in which $\xi=S \otimes \omega$ is in the "right-hand column" of $Y(g, d)$; the only such elements that are cycles are in the bottom right corner. The set of such $\xi$ is isomorphic to $\Lambda^{2 g-2-d} H^{1}\left(\Sigma_{g-1}\right)$, and because $S$ is considered to have degree 1 , the contribution to the homology from $X_{0}$ is

$$
\Lambda^{2 g-2-d} H^{1}\left(\Sigma_{g-1}\right)_{(g-1-d)} .
$$

Turning to $X_{1}$, one checks that the group of cycles is generated by elements of the form $c_{2} \otimes \omega \otimes U^{i}-c_{3} \otimes \omega \otimes U^{i}$. (The only possible case in which more cycles might appear is in the bottom left corner, but that group contains no elements of $X_{1}$.) There are no boundaries, and one can check that the group spanned by elements of the given form is isomorphic to $X(g-1, d-1)[-1]$. Together with the previous work, this gives:

Corollary 5.8 The Floer homology of $M\left(t_{\gamma}^{m} t_{\delta}^{n}\right)$ with $m \cdot n<0$ in the spin ${ }^{c}$ structures $\mathfrak{t}_{0,0, k}$, with $k \neq 0$ is given as a group by

$$
H F^{+}\left(M\left(t_{\gamma}^{m} t_{\delta}^{n}\right), \mathfrak{t}_{0,0, k}\right)=X(g-1, d-1)[-1] \oplus \Lambda^{2 g-d-2} H^{1}\left(\Sigma_{g-1}\right)_{(g-1-d)}
$$

where $d=g-1-|k|$. 
We conclude this section by pointing out that our discussions have focused on the case $k>0$. The fact that the results of theorems [5.3 and 5.7 only depend on $|k|$ follows from equation (11) together with the observation that $\mathfrak{t}_{a, b,-k}=\overline{\mathfrak{t}_{m-a, n-b, k}}$ (see also the comment after theorem 4.3).

\section{Dehn twists along a separating curve}

We now turn to the case of a mapping torus $M\left(t_{\sigma}^{ \pm 1}\right)$ obtained from a diffeomorphism of $\Sigma_{g}$ induced by a right-handed Dehn twists around a curve $\sigma \subset \Sigma_{g}$ that separates $\Sigma_{g}$ into two components of genera $g_{1}=1$ and $g_{2} \geq 1$. For convenience, we focus on the case of a right-handed Dehn twist first; the case of a left-handed twist is entirely analogous. The surgery picture for $M\left(t_{\sigma}\right)$ is

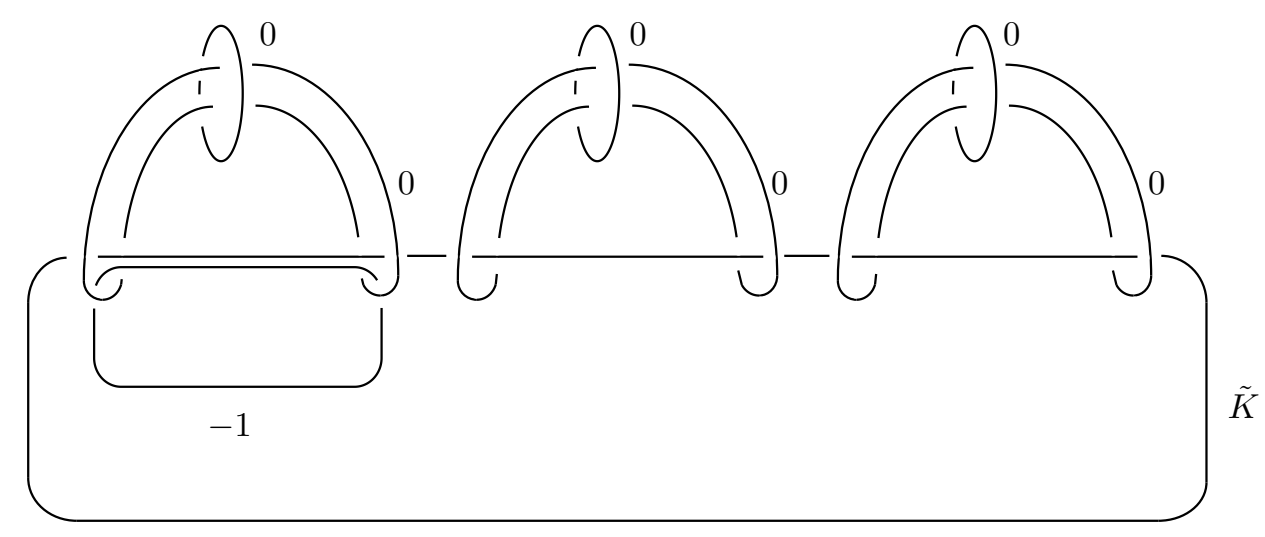

Figure 2: The knot $\tilde{K}$ in the case $g=3$.

obtained from the usual picture for $\Sigma_{g} \times S^{1}$ by adding the curve $\sigma$ with framing -1 . Figure 2 depicts the case $g=3$.

Thus the mapping torus $M\left(t_{\sigma}\right)$ can be seen as the result of 0-framed surgery on a knot $\tilde{K}$ (the "long" circle in figure 21) given by

$$
\tilde{K}=K \#^{g-1} B(0,0)
$$

where $K$ is shown in figure 3 .

Following our usual procedure, we calculate the knot Floer homology of $\tilde{K}$. To do so, it it follows from the connect sum theorem for $\widehat{H F K}$ that we need only calculate $\widehat{H F K}(M, K)$, where the 3 -manifold $M$ is the result of performing -1 surgery on one component of the Borromean rings and 0 -surgery on the other two components. 


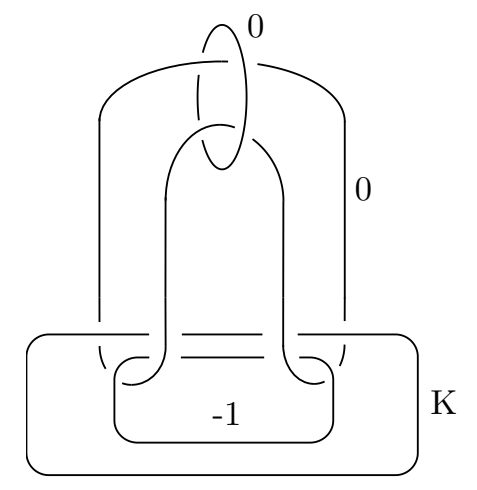

Figure 3: The knot $K$ inside $M=M\{0,0,-1\}$.

The homology $\widehat{H F}(M)$ has been calculated in [8] (where our $M$ has been denoted by $M\{0,0,-1\})$ : $\widehat{H F}(M)=\mathbb{Z}_{(0)}^{2} \oplus \mathbb{Z}_{(1)}^{2}$. We use these groups as in input for calculating the knot Floer homology for $(M, K)$.

Lemma 6.1 The knot Floer homology groups for $(M, K)$ are given by

$$
\widehat{H F K}(M, K ; j)= \begin{cases}\mathbb{Z}_{(1)} & j=1 \\ \mathbb{Z}_{(0)}^{3} \oplus \mathbb{Z}_{(1)} & j=0 \\ \mathbb{Z}_{(-1)} & j=-1 \\ 0 & \text { otherwise }\end{cases}
$$

The spectral sequence that calculates $\widehat{H F}(M)$ from $\widehat{H F K}(M, K)$ collapses at the $E_{2}$ level, and the only nontrivial differential is a surjection $d_{1}: \mathbb{Z}_{(0)}^{3} \rightarrow$ $\mathbb{Z}_{(-1)}$.

Proof We adopt the notation from [2] where $M\{p, q, r\}$ is the manifold gotten by surgery on the Borromean rings with surgery coefficients $p, q, r$. Notice that $M=M\{0,0,-1\}$.

We write the sequence in knot Floer homology arising from the triple

$$
(M\{0,0, \infty\}, B(0,0)) \rightarrow(M\{0,0,-1\}, K) \rightarrow(M\{0,0,0\}, U)
$$

where $U$ denotes the unknot in $M\{0,0,0\}=T^{3}$ (since if we replace the -1 circle in $M$ by a 0 -circle, the knot $K$ slides away from the rest of the diagram). 
This sequence appears as:

$$
\begin{array}{ccccc}
\widehat{H F K}(M\{0,0, \infty\}, B(0,0)) & \stackrel{F_{1}}{\rightarrow} \widehat{H F K}(M\{0,0,-1\}, K) & \rightarrow \widehat{H F K}\left(T^{3}, U\right) \\
\mathbb{Z}_{(1)}^{2} & & \mathbb{Z}_{(1)} & \\
\mathbb{Z}_{(-1)} & \mathbb{Z}_{(-1)} & A_{(1)} & \mathbb{Z}_{(-1 / 2)}^{3} & \mathbb{Z}_{(1 / 2)}^{3}
\end{array}
$$

Here the groups $A$ and $B$ are unknown. The arrays of groups above are arranged so that the horizontal coordinate corresponds to the absolute grading of the group and the vertical coordinate is its filtration level; the maps between arrays preserve the filtration. The map from the first to the second arrays preserves absolute grading, while the maps from the second to third and from the third to the first both decrease absolute grading by $1 / 2$.

Since for any $(Y, K)$ the knot homology $\widehat{H F K}(Y, K)$ is the $E_{1}$ term in a spectral sequence associated to a filtration of $\widehat{C F}(Y)$, there is a boundary map $d_{1}$ in each of the above arrays mapping one step diagonally down and to the left. Since $d_{1}$ is induced from the ordinary boundary in $C F$, the homomorphisms in the long exact sequences are chain maps for $d_{1}$ (they are maps of spectral sequences). Furthermore, since when we forget the filtration we have $\widehat{H F K}(M\{0,0, \infty\}, B(0,0))=\widehat{H F}(M\{0,0, \infty\})$ and $\widehat{H F K}\left(T^{3}, U\right)=\widehat{H F}\left(T^{3}\right)$, the $d_{1}$ boundaries must be trivial in those groups.

The differentials $d_{i}, i \geq 2$ in the middle term must be trivial for dimensional reasons. The homology of $d_{1}$ must therefore give $\widehat{H F}(M\{0,0,-1\})$, and it follows from the calculation quoted above that this is $\mathbb{Z}_{(1)}^{2} \oplus \mathbb{Z}_{(0)}^{2}$. In particular, $d_{1}: B \rightarrow \mathbb{Z}$ must be surjective in order that the homology vanish in degree -1 ; therefore the group $A$ can have rank 1 or 2 and has rank 1 if and only if $d_{1}: \mathbb{Z} \rightarrow B$ is trivial. We claim that this boundary map is in fact trivial.

To see this, note that $F_{1}$ is an isomorphism in filtration level 1. Furthermore, $d_{1}=0$ in $\widehat{H F K}(M\{0,0, \infty\}, B(0,0))$, so that $F_{1} \circ d_{1}=d_{1} \circ F_{1}=0$. These two facts combine to imply that $d_{1}: \mathbb{Z} \rightarrow B$ must vanish. The lemma follows.

It will be convenient in what follows to write $\widehat{H F K}(M, K)$ as

$$
\widehat{H F K}(M, K) \cong \Lambda^{*} H^{1}\left(\Sigma_{1}\right) \oplus H^{*}\left(S^{1}\right)
$$

In this notation, we can express the single nontrivial differential in the spectral sequence for $\widehat{H F}(M)$ as the map $\Lambda^{1} H^{1}\left(\Sigma_{1}\right) \rightarrow \Lambda^{0} H^{1}\left(\Sigma_{1}\right)$ given by contraction with a generator $\gamma$ of $H_{1}\left(\Sigma_{1}\right)$, which we represent as an embedded circle in the torus also denoted $\gamma$.

The connected sum theorem for $\widehat{H F K}$ then gives: 
Proposition 6.2 The knot Floer homology of $\tilde{K} \subset Y=M^{2 g-2}\left(S^{1} \times S^{2}\right)$ is given by

$$
\widehat{H F K}(Y, \tilde{K})=\Lambda^{*} H^{1}\left(\Sigma_{g}\right) \oplus\left[\Lambda^{*} H^{1}\left(\Sigma_{g-1}\right) \otimes H^{*}\left(S^{1}\right)\right] .
$$

Note that in the notation of definition 2.4, we have in this case that $M-m=1$, where in all previous cases we had $M=m$.

Under the splitting $\Sigma_{g}=\Sigma_{1} \# \Sigma_{g-1}$ and corresponding decomposition

$$
\Lambda^{*} H^{1}\left(\Sigma_{g}\right)=\Lambda_{+}^{*} H^{1}\left(\Sigma_{g}\right) \oplus \Lambda_{-}^{*} H^{1}\left(\Sigma_{g}\right)
$$

as in section [3] we have that the $d_{1}$ differential in the spectral sequence for $\widehat{H F}(Y)$ induced by $\tilde{K}$ is given by contraction with $\gamma$ on the factor $\Lambda_{-}^{*} H^{1}\left(\Sigma_{g}\right)$ in the first summand of (26), and trivial on all other factors. It is not hard to see that the homology of $d_{1}$ is equal to $\widehat{H F}(Y)$, so the spectral sequence must collapse at the $E_{2}$ stage.

We return with the above information to the calculation of $\mathrm{HF}^{+}\left(M\left(t_{\sigma}\right)\right)$. It is a simple matter to check using the results above that $H F_{r e d}^{+}(Y)=H F_{r e d}^{+}\left(Y_{p}\right)=0$ for $p>0$ sufficiently large (recall that $\left.Y=M \#(2 g-2) S^{1} \times S^{2}\right)$, so the formalism from section 2 applies. As in previous calculations, we have that $H F^{+}\left(M\left(t_{\sigma}\right) ; k\right)$ is computed by a spectral sequence whose $E_{2}$ term is the homology of $Y(g, d)$ (see (5) ) with respect to a differential constructed from the differential on $\widehat{H F K}(Y, \tilde{K})$. Here as usual, $d=g-1-|k|$ and $k \neq 0$. To describe the differential on $Y(g, d)$, note that (5) together with (26) give

$$
Y(g, d)=X(g, d) \oplus\left[X(g-1, d-1) \otimes H^{*}\left(S^{1}\right)\right] .
$$

This, together with the results of proposition 6.2 and the discussion on the differential in $Y(g, d)$ from section 3 . easily show that the the differential $D$ on $Y(g, d)$ is given by $D_{\gamma}^{+}$(see (9) ) on the factor $X(g, d)$ and is trivial elsewhere. It follows immediately that

$$
H_{*}(Y(g, d), D)=H_{*}\left(X(g, d), D_{\gamma}^{+}\right) \oplus\left[X(g-1, d-1) \otimes H^{*}\left(S^{1}\right)\right] .
$$

A simple argument shows that there can be no higher differentials in the spectral sequence calculating $H_{*}(C\{i<0$ and $j \geq k\})$, so according to Theorem $2.1 \mathrm{HF}^{+}\left(M\left(t_{\sigma}\right) ; k\right)$ is given by (27). The homology $H_{*}\left(X(g, d), D_{\gamma}^{+}\right)$was calculated in section 3 and this with similar calculation in the case $n<0$ leads to: 
Theorem 6.3 The Floer homology $\mathrm{HF}^{+}\left(M\left(t_{\sigma}^{n}\right), \mathfrak{s}_{k}\right)$ of the mapping torus $M\left(t_{\sigma}^{n}\right)$ of a right-handed $(n=1)$ or left-handed $(n=-1)$ Dehn twist around a genus 1 separating curve $\sigma$ in the surface $\Sigma_{g}(g \geq 2)$ in the Spin ${ }^{c}$ structure $\mathfrak{s}_{k}$ whose first Chern class evaluates to $2 k$ on $\left[\Sigma_{g}\right]$ and is trivial on all classes represented by tori is given by the right hand side of (27). More explicitly,

$$
\begin{aligned}
& H F^{+}\left(M\left(t_{\sigma}^{n}\right), \mathfrak{s}_{k}\right)= \\
& \quad\left(X(g-1, d-1) \otimes H^{*}\left(S^{1} \sqcup S^{1}\right)\right)[\varepsilon(n)] \oplus \Lambda^{2 g-2-d} H^{1}\left(\Sigma_{g-1}\right)_{(g-d)}
\end{aligned}
$$

with $\varepsilon(1)=0$ and $\varepsilon(-1)=-1$. Here we assume $k \neq 0$ and set $d=g-1-|k|$. If in addition $g<3 k+1$, the above isomorphisms are $\Lambda^{*} H_{1}(Y) \otimes_{\mathbb{Z}} \mathbb{Z}[U]$ module isomorphisms.

\section{References}

[1] Eaman Eftekhary, Floer cohomology of certain pseudo-Anosov maps, arXiv:math.SG/0205029

[2] Ronald Fintushel and Ronald Stern, Using Floer's exact triangle to compute Donaldson invariants, The Floer memorial volume, Progr. Math. 133, Birkhäuser 1995, 435-444. MathReview

[3] Michael Hutchings and Michael Sullivan, The periodic Floer homology of a Dehn twist, preprint at http://math.berkeley.edu/ hutching/

[4] I. G. Macdonald, Symmetric products of an algebraic curve, Topology 1 (1962), 319-343. MathReview

[5] C. McMullen, The Alexander polynomial of a 3-manifold and the Thurston norm on cohomology, Ann. Scient. École Norm. Sup. 35 (2000), 153-171. MathReview

[6] G. Meng and C. H. Taubes, $\underline{S W}=$ Milnor torsion, Math. Res. Lett. 3 (1996), 661-674. MathReview

[7] Dean Neumann, 3-manifolds fibering over $S^{1}$, Proc. Amer. Math. Soc. 58 (1976), 353-356. MathReview

[8] Peter Ozsváth and Zoltán Szabó, Absolutely graded Floer homologies and intersection forms for four-manifolds with boundary, Adv. Math. 173 (2003), 179-261. MathReview

[9] Peter Ozsváth and Zoltán Szabó, Holomorphic disks and knot invariants, Adv. Math. 186 (2004), 58-116. MathReview

[10] Peter Ozsváth and Zoltán Szabó, Holomorphic disks and three-manifold invariants: properties and applications, arXiv:math.SG/0105202

[11] Peter Ozsváth and Zoltán Szabó, Holomorphic disks and topological invariants for closed 3-manifolds, arXiv:math.SG/0101206 
[12] Peter Ozsváth and Zoltán Szabó, Holomorphic triangles and invariants for smooth four-manifolds, arXiv:math.SG/0110169

[13] Peter Ozsváth and Zoltán Szabó, Holomorphic triangle invariants and the topology of symplectic four-manifolds, Duke Math. J. 121 (2004), 1-34. MathReview

[14] Paul Seidel, The symplectic Floer homology of a Dehn twist, Math. Res. Lett. 3 (1996), 829-834. MathReview

[15] William Thurston, A norm for the homology of 3-manifolds, Mem. Amer. Math. Soc. 59 (1986), No. 339, 99-130. MathReview

[16] Jeffrey Tollefson, 3-manifolds fibering over $S^{1}$ with nonunique connected fiber, Proc. Amer. Math. Soc. 21 (1969), 79-80. MathReview

Department of Mathematics, Columbia University

2990 Broadway, New York, NY 10027, USA

and

Department of Mathematics, Southeastern Louisiana University

1205 North Oak Street, Hammond, LA 70402, USA

Email: jabuka@math.columbia.edu and Thomas.Mark@selu.edu

Received: 6 July 2004 